\title{
Evolution of hypersurfaces by powers of the scalar curvature
}

\author{
Roberta Alessandroni And CARlo Sinestrari
}

\begin{abstract}
We study the evolution of a closed hypersurface of the euclidean space by a flow whose speed is given by a power of the scalar curvature. We prove that, if the initial shape is convex and satisfies a suitable pinching condition, the solution shrinks to a point in finite time and converges to a sphere after rescaling. We also give an example of a nonconvex hypersurface which develops a neckpinch singularity.
\end{abstract}

Mathematics Subject Classification (2010): 53C44 (primary); 35K55, 58J35, 35B40 (secondary).

\section{Introduction}

In this work we study the behaviour of a convex hypersurface of the Euclidean space moving by powers of the scalar curvature. In particular, we find hypotheses under which the so called "convergence to a round point" holds, that is, the hypersurface shrinks to a point and converges to a sphere after rescaling.

The convergence to a round point was first proved for convex hypersurfaces evolving by the mean curvature flow by Huisken [14]. Since then, many authors have studied the same problem for flows where the speed is given by other symmetric homogeneous functions $\mathcal{S}$ of the principal curvatures. For instance, B. Chow has considered the cases $\mathcal{S}=\sqrt{R}$, where $R$ is the scalar curvature [10], and $\mathcal{S}=K^{\beta}$, with $\beta \geq 1 / n$, where $K$ is the Gauss curvature [9]. More recently, B. Andrews proved convergence to a round point for a wide class of speeds homogeneous of degree one (see $[2,6,7])$. When the degree is greater than one, less results are known: the papers in the literature concern particular flows and are often restricted to surfaces of dimension two, see $[3,9,20,22]$. The case where the degree is less than one is even more difficult. In some case it is known that convex hypersurfaces shrink to a point $[4,21]$, but some counterexamples show that in general the profile does not necessarily become spherical after rescaling.

The case we consider in this paper corresponds to $\mathcal{S}=R^{p}$ with $p>1 / 2$. For this choice of powers the speed is a homogeneous function of the principal

The authors have been partially supported by the Italian project PRIN 2007WECYEA.

Received June 17, 2009; accepted September 16, 2009. 
curvatures of degree greater than one. To state our main theorem it is useful to introduce the function $f=\frac{n-1}{n}-\frac{R}{H^{2}}$, where $H$ is the mean curvature. We have the identity

$$
f H^{2}=\frac{1}{n} \sum_{i<j}\left(\lambda_{i}-\lambda_{j}\right)^{2},
$$

where $\lambda_{i}$ are the principal curvatures, which shows that $f$ is a nonnegative function measuring how much the curvatures differ from each other. Our main theorem is the following:

Theorem 1.1. Let $\mathbf{F}_{0}: M \longrightarrow \mathbb{R}^{n+1}$ be a smooth embedding of a closed $n$ dimensional manifold, with $n \geq 2$, such that $M_{0}=\mathbf{F}_{\mathbf{0}}(M)$ is uniformly convex. Then the initial value problem

$$
\begin{cases}\frac{\partial}{\partial t} \mathbf{F}(\mathbf{p}, t)=-R^{p}(\mathbf{p}, t) v(\mathbf{F}(\mathbf{p}, t)) & \mathbf{p} \in M, t \geq 0 \\ \mathbf{F}(\mathbf{p}, 0)=\mathbf{F}_{0}(\mathbf{p}) & \mathbf{p} \in M,\end{cases}
$$

with $p>1 / 2$, has a unique smooth solution in a finite time interval $[0, T)$. If the initial surface $M_{0}$ satisfies at every point the pinching estimate $f \leq \gamma^{*}(n, p)$ for a suitable constant $\gamma^{*}(n, p)$ (given in Definition 5.3), then the surfaces $M_{t}=$ $\mathbf{F}(M, t)$ are convex for all $t$ and converge to a single point $\mathbf{y}_{T}$ as $t \rightarrow T$.

Moreover, if we define the rescaled immersions and the new time parameter

$$
\widetilde{\mathbf{F}}(\mathbf{p}, t)=\left(c^{\prime}(T-t)\right)^{-\frac{1}{2 p+1}}\left(\mathbf{F}(\mathbf{p}, t)-\mathbf{y}_{T}\right), \quad \tau=-\frac{1}{c^{\prime}} \ln \left(1-\frac{t}{T}\right)
$$

where $c^{\prime}=n^{p}(n-1)^{p}(2 p+1)$, we have that $\underset{\widetilde{\mathbf{F}}}{\widetilde{T}}(\cdot, \tau) \rightarrow \widetilde{\mathbf{F}}_{\infty}$ as $\tau \rightarrow+\infty$ in the $C^{\infty}$ topology with exponential speed, where $\widetilde{\mathbf{F}}_{\infty}$ is a smooth embedding of a unit sphere.

The main step in the proof of this theorem consists of showing that the maximum of $f$ is decreasing in time, and that the same property holds also for the function $f_{\sigma}=f H^{\sigma}$ for $\sigma>0$ small enough. The monotonicity of $f$ is used to show that the convexity and the pinching of the curvatures are preserved by the flow. The estimate on $f_{\sigma}$ implies that $f$ tends to zero at those points where $H$ becomes unbounded; thus, the shape of our hypersurface becomes spherical when a singularity is approached.

We also show in this paper that our flow is well posed for initial hypersurfaces with positive scalar curvature, and that positive scalar curvature is preserved under the flow. In addition, we prove that for any $n \geq 3$ there exist nonconvex dumbbellshaped hypersurfaces developing a neckpinch singularity similar to the case of the mean curvature flow.

As recalled above, this flow has been studied in the case $p=1 / 2$ by $\mathrm{B}$. Chow in [10], who proved convergence to a round point if the initial hypersurface satisfies 
a pinching condition similar to ours. Recently, B. Andrews has extended this result to any convex initial data, as a particular case of a theorem in [7]. Our results for $p>1 / 2$ are new, except for the case $n=2$; in this dimension the scalar curvature coincides with the Gauss curvature and some convergence results for $p>1 / 2$ have already been obtained in $[6,9]$. In particular, it is known that in the case $n=2, p=1$ the convergence to a round point holds for all convex surfaces [3].

The degree of homogeneity greater than one in the speed of our flow changes some features of the problem with respect to the case $p=1 / 2$. One main difference is that the gradient terms in the evolution equation for the pinching function $f$ have a worse behaviour, and they can only be estimated if a condition of the form $f \leq \gamma^{*}$ is satisfied, as in the assumption of our theorem. A similar condition has been assumed in the papers $[9,22]$ that have dealt with other flows with homogeneity greater than one. Here we also provide an explicit expression of the pinching constant $\gamma^{*}$, in order to discuss its dependence on $n, p$ (see Remark 5.4). Another problem related to the higher degree of homogeneity arises in the analysis of the rescaled flow, where the evolution equations become degenerate parabolic. To deal with this difficulty we adapt a procedure developed by Schulze in [22], which is based on a Hölder estimate by Di Benedetto and Friedman [11]. On the other hand, a good feature related to the higher degree of homogeneity is the presence of a nicely behaved reaction term in the equation satisfied by $f$. This allows to bound from above the function $f_{\sigma}=f H^{\sigma}$ by a direct application of the maximum principle, without using integral estimates and iteration techniques as in $[10,14]$.

A natural conjecture is that the statement of our theorem holds for any initial convex hypersurface without assuming the pinching condition. However, the discussion in [6] suggests that the pinching of the curvatures cannot be monotone on a general convex hypersurface if the speed of the flow is homogeneous with a degree greater than one. Therefore, different methods from the ones used here are needed. Until now, however, this has been obtained only for certain flows in dimension $n=2[3,20]$; for a general dimension, it remains an open problem.

Let us finally mention that flows similar to the one considered here, but in a lorentzian ambient manifold, have been studied in $[8,13]$ to prove the existence of hypersurfaces with prescribed scalar curvature. In these papers suitable barriers are constructed, which ensure the long time existence of the flow and the convergence to a limit hypersurface with the desired properties.

The paper is organized as follows. After fixing some notation in Section 2, we prove the short time existence and regularity of the solution to (1.1) in Section 3. Then in Section 4 we write the evolution equations for the main geometric quantities associated to the surfaces $M_{t}$. Section 5 is devoted to the proof of the monotonicity of the pinching function $f$, whereas the following sections 6 and 7 concern the convergence to a round point. Finally, in Section 8 we give an example of a nonconvex hypersurface developing a neckpinch singularity.

ACKnOwledgements. During their work the authors have visited the AEI in Golm; they thank the Institute for the hospitality and are grateful to G. Huisken, O. Schnürer and F. Schulze for useful discussions. 


\section{Notation}

Given an $n$-dimensional closed, orientable manifold $M$ and a smooth embedding in Euclidean space $\mathbf{F}: M \rightarrow \mathbb{R}^{n+1}$, we denote by $g$ the metric on $\mathbf{F}(M)$ induced by the standard scalar product $\langle\cdot, \cdot\rangle$ of $\mathbb{R}^{n+1}$. The inverse matrix of $g$ is denoted by $g^{*}$. As usual, we denote by $g_{i j}$ and $g^{i j}$ the components of $g$ and $g^{*}$ respectively in a given local coordinate system. The matrices $g_{i j}$ and $g^{i j}$ are also used to lower and raise the indices of the tensors. Throughout the paper we use the Einstein summation convention.

We use the letter $\mathbf{p}$ for the generic point on $M$, and $\mathbf{x}, \mathbf{y}$ for the points in $\mathbb{R}^{n}$ and $\mathbb{R}^{n+1}$ respectively. We denote by $v$ the outer normal vector on $\mathbf{F}(M)$ and consider the second fundamental form

$$
\mathcal{I I}(\mathbf{p}): T_{\mathbf{p}} \mathbf{F}(M) \times T_{\mathbf{p}} \mathbf{F}(M) \rightarrow \mathbb{R}
$$

whose elements are $h_{i j}(\mathbf{p})=\left\langle\frac{\partial \mathbf{F}}{\partial x_{i}}(\mathbf{p}), \frac{\partial v}{\partial x_{j}}(\mathbf{p})\right\rangle=-\left\langle\frac{\partial^{2} \mathbf{F}}{\partial x_{i} \partial x_{j}}(\mathbf{p}), v(\mathbf{p})\right\rangle$. The elements of the Weingarten map $\mathcal{W}: T_{\mathbf{p}} \mathbf{F}(M) \rightarrow T_{\mathbf{p}} \mathbf{F}(M)$ are $h_{i}^{j}=h_{i k} g^{k j}$ and its eigenvalues, called principal curvatures, are denoted as $\left(\lambda_{1}, \ldots, \lambda_{n}\right)$ with $\lambda_{1} \leq \ldots \leq \lambda_{n}$.

If the surface $\mathbf{F}(M)$ is written locally as a graph $\mathbf{F}(\mathbf{p})=:(\mathbf{x}, u(\mathbf{x}))$, then we have

$$
g_{i j}(\mathbf{p})=\delta_{i j}+D_{i} u(\mathbf{x}) D_{j} u(\mathbf{x}), \quad g^{i j}(\mathbf{p})=\delta^{i j}-\frac{D_{i} u(\mathbf{x}) D_{j} u(\mathbf{x})}{1+|D u(\mathbf{x})|^{2}} .
$$

Moreover if the outer normal vector points below then

$$
v(\mathbf{p})=\frac{(D u(\mathbf{x}),-1)}{\sqrt{1+|D u(\mathbf{x})|^{2}}}
$$

while the elements of the second fundamental form and the Christoffel symbols satisfy:

$$
h_{i j}(\mathbf{p})=\frac{D_{i j}^{2} u(\mathbf{x})}{\sqrt{1+|D u(\mathbf{x})|^{2}}} \quad \text { and } \quad \Gamma_{i j}^{k}(\mathbf{p})=\frac{D_{i j}^{2} u(\mathbf{x}) D_{k} u(\mathbf{x})}{1+|D u(\mathbf{x})|^{2}} .
$$

From now on we use the following notation: the mean curvature is $H=\operatorname{Tr}(\mathcal{W})=$ $\lambda_{1}+\cdots+\lambda_{n}$, the squared norm of the second fundamental form is $|A|^{2}$ $=\operatorname{Tr}\left(\mathcal{W} \mathcal{W}^{T}\right)=\lambda_{1}^{2}+\cdots+\lambda_{n}^{2}$ and the scalar curvature is

$$
R=H^{2}-|A|^{2}=2 \sum_{i<j} \lambda_{i} \lambda_{j}
$$

Let us consider a smooth hypersurface immersion $\mathbf{F}_{0}: M \rightarrow \mathbb{R}^{n+1}$ as described before, with $n \geq 2$ and let $\mathbf{F}: M \times[0, T) \rightarrow \mathbb{R}^{n+1}$ be the one parameter family of 
immersions defined by the initial value problem (1.1). For all $t \in[0, T)$ we set $\mathbf{F}_{t}=$ $\mathbf{F}(\cdot, t)$ and $M_{t}=\mathbf{F}_{t}(M)$. In what follows we consider on every hypersurface $M_{t}$ the quantities defined above without referring explicitly to their dependence on point and time. For simplicity, we often use the word "surface" instead of "hypersurface" to denote our evolving manifold, also if $n>2$.

As in [2], we denote by $\nabla$ the covariant derivative on $M_{t}$ and by $\mathrm{Hess}_{\nabla}$ the second derivative as a 2-covariant tensor. If $\mathrm{Hess}_{\nabla}$ is contracted with the standard metric $g$ it gives the standard Laplace Beltrami operator. More in general, if $m=$ $\left\{m_{i j}\right\}$ is another metric then we can associate to $m$ the elliptic operator

$$
\Delta_{m} T=\mathcal{M} g^{*}\left(\operatorname{Hess}_{\nabla} T\right)=m^{i j} \nabla_{i} \nabla_{j} T .
$$

We use a similar notation for the inner product induced by a 2-covariant tensor: given a symmetric bilinear form $b_{i j}$ we set $\left\langle T_{i}, V_{i}\right\rangle_{b}=T_{i} b^{i j} V_{j}$ and $\left|T_{i}\right|_{b}^{2}=\left\langle T_{i}, T_{i}\right\rangle_{b}$. If the tensors $T$ and $V$ have more than one index, then the bilinear form $b_{i j}$ is used only to contract the index $i$, while the other ones are contracted with the metric tensor $g_{i j}$.

\section{Existence results}

In this section we prove the short time existence of the solution to (1.1) if the initial hypersurface has positive scalar curvature and we give a regularity theorem ensuring that the evolving surfaces $M_{t}$ 's remain smooth as long as the principal curvatures are bounded. Here and in the next section the exponent $p$ in the speed of our flow can be any positive value.

We need the following elementary remark: if $M$ is a closed surface, then

$$
R>0 \text { everywhere on } M \Longrightarrow H>0 \text { everywhere on } M \text {. }
$$

In fact, if $R$ is positive everywhere, then $H^{2}=R+|A|^{2}$ is also positive. Therefore $H$ has constant sign, otherwise we would have $H^{2}=0$ somewhere. Since on a closed surface there is at least one point with $H>0$, the sign of $H$ has to be positive. We also recall the following property, valid on any given point $\mathbf{p} \in M$, (see e.g. [17, Lemma 2.4]):

$$
H>0, R>0 \Longrightarrow H-\lambda_{k}>0, \quad \forall k=1, \ldots, n .
$$

Theorem 3.1. Let $M_{0}$ be a closed hypersurface with positive scalar curvature. Then the initial value problem (1.1) has a unique smooth solution at least for a short time interval $[0, T)$.

Proof. It is well known (see [15, Theorem 3.1]) that the small time existence for a flow driven by a speed $\mathcal{S}$, with $\mathcal{S}$ a symmetric function of the curvatures, is ensured provided the condition

$$
\frac{\partial \mathcal{S}}{\partial \lambda_{k}}>0 \quad \forall k=1, \ldots, n
$$


holds at every point of the initial surface. Such a property holds in our case, because we have

$$
\frac{\partial R^{p}}{\partial \lambda_{k}}=2 p R^{p-1}\left(H-\lambda_{k}\right)
$$

and the right-hand side is positive by (3.1) and (3.2).

We now want to show that the solution remains smooth as long as the curvature are bounded. To prove this, we use the following result (see [5, Theorem 6]):

Theorem 3.2. Let $\Omega$ be a domain of $\mathbb{R}^{n}$. Let $u \in C^{4}(\Omega \times[0, T))$ a function satisfying

$$
\frac{\partial u}{\partial t}=G\left(D^{2} u, D u\right)
$$

where $G \in C^{2}$ is elliptic, i.e., there exist two constants $\Lambda_{1}$ and $\Lambda_{2}$ such that $0<$ $\Lambda_{1} I \leq \dot{G} \leq \Lambda_{2} I$, where $\dot{G}$ is the matrix of the partial derivatives with respect to $D^{2} u$. Suppose that $G$ can be written as $G\left(D^{2} u, D u\right)=\phi\left(L\left(D^{2} u, D u\right)\right)$, where $\phi$ $i$ such that $\phi^{\prime} \neq 0$ on the range of $L$ and $L$ is concave with respect to $D^{2} u$. Then in any relatively compact set $\Omega^{\prime} \subset \Omega$ and for any $\tau \in(0, T)$ we have

$$
\|u\|_{C^{2, \alpha}\left(\Omega^{\prime} \times[\tau, T)\right)} \leq K,
$$

where $K$ depends on $\Lambda_{1}, \Lambda_{2},\|u\|_{C^{2}(\Omega \times[0, T))}$, $\tau$, dist $\left(\Omega^{\prime}, \partial \Omega\right)$ and the bounds on the first and second derivatives of $G$.

The interest of the above theorem is that it allows to relax the concavity hypothesis of the usual regularity theorem for fully nonlinear parabolic equations (see e.g. [18, 19]).

Theorem 3.3. Let $M_{t}$ be a solution of the flow (1.1) defined for $t \in[0, T)$. Suppose that $|A|^{2}$ is uniformly bounded, and that $R$ is greater than some positive constant on $M_{t}$ for all $t \in[0, T)$. Then any derivative of $A$ is also uniformly bounded for $t \in[0, T)$.

Proof. Let us take any point $\left(\mathbf{p}_{0}, t_{0}\right) \in M \times[0, T)$ and set $\mathbf{y}_{0}=\mathbf{F}_{t_{0}}\left(\mathbf{p}_{0}\right)$. Consider the tangent plane $T_{\mathbf{p}_{0}} M_{t_{0}}$ to our surface at that point. Then, for $\varepsilon$ suitably small, the surface $M_{t}$ can be locally written as the graph of a function $u(\mathbf{x}, t)$ with $\mathbf{x} \in \Omega=$ $B_{2 \varepsilon}\left(\mathbf{y}_{0}\right) \cap T_{\mathbf{p}_{0}} M_{t_{0}}$ and $t \in\left[t_{0}-\varepsilon, t_{0}+\varepsilon\right]$. Note that, since the principal curvatures of $M_{t}$ are uniformly bounded, we can choose a uniform $\varepsilon>0$ such that the above can be done for all $\mathbf{p}_{0} \in M$ and $t_{0} \in[\varepsilon, T-\varepsilon)$, and such that the $C^{2}$ norm of $u$ is bounded by the same constant for the graph representation around any point. Here we use also the correspondence between the second fundamental form $h_{i j}$ of $M_{t}$ and the Hessian $D_{i j}^{2} u$ of $u$ recalled in the previous section.

By (2.1) and (2.3), the scalar curvature of $M_{t}$ in these local coordinates has the form $R=\widetilde{R}\left(D^{2} u, D u\right)$ for a suitable function $\widetilde{R}$. In addition, $u$ evolves according to

$$
\frac{\partial u}{\partial t}=-\sqrt{1+|D u|^{2}}\left\langle\frac{\partial}{\partial t} \mathbf{F}, v\right\rangle=\widetilde{R}^{p}\left(D^{2} u, D u\right) \sqrt{1+|D u|^{2}} .
$$


To check that the right hand side of the above equation satisfy the requirements of Theorem 3.2, we use some general facts about the functions of the eigenvalues of a matrix. In fact, the property that $\partial R^{p} / \partial \lambda_{i}>0$ for every $i$ implies the ellipticity of $\widetilde{R}^{p}$ as a function of $D^{2} u$. Since our surfaces have positive scalar curvature, properties (3.1) and (3.2) allow to show that our operator is uniformly elliptic. We then recall that $\sqrt{R}$ is a concave function of $\lambda_{1}, \ldots, \lambda_{n}$ (see e.g. [19, Theorem 15.17]). This implies (see e.g. [7, Corollary 5.3]) that $\sqrt{\widetilde{R}}$ is a concave function of $D^{2} u$. Therefore, the right hand side of (3.3) can be written as the $2 p$-power of a concave function. By Theorem 3.2 we derive a $C^{2, \alpha}$ estimate on $u$.

Once such an estimate is established, standard parabolic theory allows to derive bounds on $\|u\|_{C^{k}}$ for any $k>2$, which imply estimates on any derivative of the curvature. Since these bounds do not depend on $\left(p_{0}, t_{0}\right)$, the theorem follows.

We will see in the following that in the previous theorem it suffices to assume the positivity of $R$ at the initial time, because the minimum of $R$ is nondecreasing under the flow.

\section{Evolution equations}

As our hypersurface evolves according to the flow (1.1), the associated geometric quantities change and satisfy suitable evolution equations that can be computed following the procedure of [14] (see also e.g. [2, 10]). It is convenient to introduce the tensor $m_{i j}:=H g_{i j}-h_{i j}$. We recall that, by (3.1)-(3.2), $m_{i j}$ is positive definite on any closed surface with positive scalar curvature. Then, by a straightforward computation we find the following result:

Proposition 4.1. Let $\mathbf{F}_{t}: M \longrightarrow \mathbb{R}^{n+1}$ be the solution to (1.1) for all $t \in[0, T)$. Then the following equations hold

$$
\frac{\partial g_{i j}}{\partial t}=-2 R^{p} h_{i j}, \quad \frac{\partial v}{\partial t}=\nabla R^{p}, \quad \frac{\partial d \mu}{\partial t}=-R^{p} H d \mu .
$$

In general, if $P$ is a homogeneous function of degree $\alpha$ in the Weingarten map $\mathcal{W}$, the evolution equation for $P$ is

$$
\begin{aligned}
\frac{\partial P}{\partial t}= & 2 p R^{p-1}\left\{\Delta_{m} P-m g^{*} g^{*} \ddot{P}(\nabla \mathcal{W}, \nabla \mathcal{W})+\dot{P} g^{*}(\nabla H, \nabla H)\right. \\
& -\dot{P} g^{*}(\nabla A, \nabla A)+\frac{p-1}{2} \frac{1}{R} \dot{P} g^{*}(\nabla R, \nabla R) \\
& \left.-\frac{2 p-1}{2 p} R \dot{P}\left(\mathcal{W}^{2}\right)+\alpha\left(H|A|^{2}-C\right) P\right\}
\end{aligned}
$$

where $C=\operatorname{tr}\left(\mathcal{W}^{3}\right)=\sum \lambda_{i}^{3}$. 
For the reader's convenience, we write equation (4.2) also in the classical notation with indices:

$$
\begin{aligned}
\frac{\partial P}{\partial t} & =2 p R^{p-1}\left\{\Delta_{m} P-m^{i j} \frac{\partial^{2} P}{\partial h_{l}^{k} \partial h_{q}^{p}} \nabla_{i} h_{l}^{k} \nabla_{j} h_{q}^{p} \frac{\partial P}{\partial h_{j}^{i}} \nabla^{i} H \nabla_{j} H\right. \\
& -\frac{\partial P}{\partial h_{j}^{i}} \nabla^{i} h_{l}^{k} \nabla_{j} h_{k}^{l}+\frac{p-1}{2} \frac{1}{R} \frac{\partial P}{\partial h_{j}^{i}} \nabla^{i} R \nabla_{j} R \\
& \left.-\frac{2 p-1}{2 p} R \frac{\partial P}{\partial h_{j}^{i}} h_{k}^{j} h_{i}^{k}+\alpha\left(H|A|^{2}-C\right) P\right\} .
\end{aligned}
$$

As an application of (4.2), we obtain:

Corollary 4.2. We have the following evolution equations:

$$
\begin{aligned}
\frac{\partial H}{\partial t}= & 2 p R^{p-1}\left\{\Delta_{m} H+|\nabla H|^{2}-|\nabla A|^{2}+\frac{p-1}{2} \frac{1}{R}|\nabla R|^{2}\right. \\
& \left.-\frac{2 p-1}{2 p} R|A|^{2}+\left(H|A|^{2}-C\right) H\right\} \\
\frac{\partial|A|^{2}}{\partial t}= & 2 p R^{p-1}\left\{\Delta_{m}|A|^{2}+2\left|\nabla_{i} H\right|_{h}^{2}-2\left|\nabla_{i} A\right|_{H g}^{2}\right. \\
& \left.+(p-1) \frac{1}{R}\left|\nabla_{i} R\right|_{h}^{2}-\frac{2 p-1}{p} R C+2\left(H|A|^{2}-C\right)|A|^{2}\right\} \\
\frac{\partial R}{\partial t}= & 2 p R^{p-1}\left\{\Delta_{m} R+(p-1) \frac{1}{R}\left|\nabla_{i} R\right|_{m}^{2}+\frac{1}{p}\left(H|A|^{2}-C\right) R\right\} \\
\frac{\partial R^{p}}{\partial t}= & 2 p R^{p-1}\left\{\Delta_{m} R^{p}+\left(H|A|^{2}-C\right) R^{p}\right\} \cdot
\end{aligned}
$$

Corollary 4.3. Let $M_{0}$ be a closed hypersurface with $R>0$, and let $M_{t}$ be its evolution under the flow (1.1), defined in a maximal time interval $[0, T)$. Then $M_{t}$ has positive scalar curvature for all $t, T$ is finite, and we have

$$
\limsup _{t \rightarrow T}\left(\max _{M_{t}}\|A\|^{2}\right)=+\infty
$$

Proof. Let us first estimate from below the term $H|A|^{2}-C$ which appears in the evolution equation for $R$ (4.3). From [16, Lemma 2.2] we have $C \leq|A|^{3}$; if we use in addition the inequalities $H^{2} \leq n|A|^{2}$ and $R \leq(n-1)|A|^{2}$ we obtain

$$
H|A|^{2}-C \geq|A|^{2}(H-|A|)=\frac{|A|^{2} R}{H+|A|} \geq \frac{|A| R}{\sqrt{n}+1} \geq \frac{R^{\frac{3}{2}}}{\sqrt{n-1}(\sqrt{n}+1)} .
$$


Now we define the function $\varphi(t):=\min _{M_{t}} R$. Using the evolution equation (4.3) for $R$ and the maximum principle we obtain that $\varphi$ satisfies in a weak sense the inequality

$$
\frac{d \varphi}{d t} \geq \frac{1}{\sqrt{n-1}(\sqrt{n}+1)} \varphi^{p+\frac{3}{2}} .
$$

This shows that $\varphi$ is increasing, and that $R$ remains positive on $M_{t}$ for $t \in(0, T)$. In addition, since $p+3 / 2>1$, a comparison with the corresponding o.d.e. shows that $\varphi$ becomes unbounded in finite time, and so the maximal time $T$ must be finite.

The last assertion can be proved by contradiction. If $|A|^{2}$ remains bounded, we can use Theorem 3.3 and a standard argument (see [14, Theorem 8.1]) to show that $M_{t}$ converges smoothly to a limit surface $M_{T}$, from which the flow can be restarted, in contradiction with the maximality of $T$.

\section{Pinching of curvatures}

To analyze the pinching of the curvatures of our evolving hypersurface, we consider the function

$$
f:=\frac{|A|^{2}}{H^{2}}-\frac{1}{n},
$$

also considered in $[10,14]$. It is easily checked that

$$
0 \leq f H^{2}=\frac{1}{n} \sum_{i<j}\left(\lambda_{i}-\lambda_{j}\right)^{2} .
$$

Hence, at any point $f$ gives a measure of how much principal curvatures differ from each other. Let us first prove some relations between the value of $f$ and the properties of the curvatures, which will be crucial in deriving the main estimates of this section.

Proposition 5.1. The following properties hold:

i) At any point where $f \leq \frac{1}{n(n-1)}-\varepsilon$, for some $\varepsilon>0$, we have $\lambda_{1} \geq \frac{n-1}{2} \varepsilon H$.

ii) We have $\lambda_{n} \leq\left(\sqrt{\frac{n-1}{n} f}+\frac{1}{n}\right) H$.

Proof. Let us isolate one eigenvalue, say $\lambda_{k}$, and write $H=H^{\prime}+\lambda_{k}$ and $|A|^{2}=$ $\left|A^{\prime}\right|^{2}+\lambda_{k}^{2}$. We find

$$
\begin{aligned}
f H^{2} & =|A|^{2}-\frac{1}{n} H^{2}=\left|A^{\prime}\right|^{2}+\lambda_{k}^{2}-\frac{1}{n} H^{\prime 2}-\frac{2}{n} H^{\prime} \lambda_{k}-\frac{1}{n} \lambda_{k}^{2} \\
& =\left(\left|A^{\prime}\right|^{2}-\frac{1}{n-1} H^{\prime 2}\right)+\frac{n}{n-1}\left(\frac{n-1}{n} \lambda_{k}-\frac{1}{n} H^{\prime}\right)^{2} \\
& \geq \frac{n}{n-1}\left(\frac{n-1}{n} \lambda_{k}-\frac{1}{n} H^{\prime}\right)^{2}=\frac{n}{n-1}\left(\lambda_{k}-\frac{1}{n} H\right)^{2} .
\end{aligned}
$$


Thus we have

$$
\sqrt{\frac{n-1}{n} f} H \geq\left|\lambda_{k}-\frac{1}{n} H\right| .
$$

In order to prove the first part we set $\lambda_{k}=\lambda_{1}$ and assume $f \leq \frac{1}{n(n-1)}-\varepsilon$. Using the inequality $\sqrt{1-a} \leq 1-a / 2$ for any $a \leq 1$ we obtain

$$
\begin{aligned}
\lambda_{1} & \geq\left(\frac{1}{n}-\sqrt{\frac{n-1}{n} f}\right) H \geq \frac{1-\sqrt{1-(n-1) n \varepsilon}}{n} H \\
& \geq \frac{(n-1) \varepsilon}{2} H .
\end{aligned}
$$

For the proof of assertion ii) we take $\lambda_{k}=\lambda_{n}$ and we find

$$
\lambda_{n} \leq\left(\sqrt{\frac{n-1}{n} f}+\frac{1}{n}\right) H \text {. }
$$

Corollary 5.2. If $f<1 / n(n-1)$ everywhere on $M$, then $M$ is uniformly convex. The converse implication holds only if $n=2$.

Proof. The first statement is a direct consequence of part (i) of the previous proposition. To see that if $n=2$ the converse holds, it suffices to observe that if $n=2$ then $\frac{1}{2}-f=2 \lambda_{1} \lambda_{2} H^{-2}$. If $n>2$, instead, the positivity of the curvatures does not imply that $f<1 / n(n-1)$. In fact, if $\lambda_{1}, \ldots, \lambda_{n-1}$ are positive, but very small compared to $\lambda_{n}$, then $|A|^{2} \approx H^{2}$ and so $f \approx 1-1 / n>1 / n(n-1)$.

The aim of this section is to prove that, if the maximum of $f$ is smaller than a suitable constant $\gamma^{*}$ on the initial surface, then it is nonincreasing in time with the flow. Furthermore, we will prove that the same holds for the function $f H^{\sigma}$ for $\sigma>0$ suitably small, showing that $f$ tends to zero as $t \rightarrow T$, at least at those points where the mean curvature diverges. We are able to prove these results for a suitable choice of $\gamma^{*}$, depending on $n, p$, which is given below.

Definition 5.3. For $n \geq 4$ and $p>1 / 2, n$ integer, we define $\gamma^{*}(n, p):=$ $\min \left\{\gamma_{1}(n), \gamma_{2}(n, p)\right\}$, where

$$
\gamma_{1}(n)=\frac{1}{n(n-1)} \quad \text { and } \quad \gamma_{2}(n, p)=\frac{4(n-1)}{n(n+2)^{2}}\left(\frac{1}{2 p-1}\right)^{2}
$$

In the case $n=3$, we define instead

$$
\gamma^{*}(3, p)= \begin{cases}\gamma_{1}(3) & \text { if } \frac{1}{2}<p \leq \frac{4}{5} \\ \frac{8}{75 p^{2}} & \text { if } \frac{4}{5} \leq p \leq 1 \\ \gamma_{2}(3, p) & \text { if } p \geq 1 .\end{cases}
$$


Finally, for $n=2$ we set

$$
\gamma^{*}(2, p)= \begin{cases}\frac{9}{2}-8 p & \text { if } \frac{1}{2}<p \leq \frac{33}{62} \\ \frac{3-2 p}{8} & \text { if } \frac{33}{62} \leq p \leq 1 \\ \gamma_{2}(2, p) & \text { if } p \geq 1 .\end{cases}
$$

Remark 5.4. The above definition is given in such a way that some estimates needed in the proofs of the next results (in particular, (5.4) in Lemma 5.6) are satisfied. In fact, we could give an alternative definition of $\gamma^{*}(n, p)$ as the largest constant which fulfills certain inequalities, and this would give a slightly better value for certain ranges of $n, p$. However, we prefer the above definition, which is explicit (although somehow elaborate in the cases $n=2,3$ ) and allows us to underline some interesting properties of this constant:

- For all values of $n, p$, we have $\gamma^{*}(n, p) \leq 1 / n(n-1)$; hence, by Corollary 5.2, the inequality $f \leq \gamma^{*}$ implies the convexity of $M$.

- For $n \geq 3$ and $p$ suitably close to $1 / 2$, we have $\gamma^{*}(n, p)=1 / n(n-1)$. This is the same constant obtained by B. Chow [10] in the case $p=1 / 2$.

- For $n=2$, we have $\gamma^{*}(2, p)>1 / 2=1 / n(n-1)$ for all $p>1 / 2$; more precisely, $\gamma^{*}(2, p) \downarrow 1 / 2$ as $p \downarrow 1 / 2$.

- For any $n \geq 2, \gamma^{*}(n, p)$ is a nonincreasing function of $p$ and it decays like $O\left(1 / p^{2}\right)$ as $p \rightarrow \infty$.

Hence, the surfaces satisfying $f \leq \gamma^{*}(n, p)$ are a strict subset of the class of convex surfaces, and the condition becomes more restrictive as the homogeneity degree of the speed increases.

As a first step in our analysis, we write the evolution equation for $f$ in a suitable form for the application of the maximum principle.

Lemma 5.5. The function $f$ satisfies the evolution equation

$$
\begin{aligned}
\frac{\partial f}{\partial t}= & 2 p R^{p-1}\left\{\Delta_{m} f-(p-1) \frac{H}{R}\left|\nabla_{i} f\right|_{|A|^{2} g-H h}^{2}+4 \frac{1}{H}\left\langle\nabla_{i} f, \nabla_{i} H\right\rangle_{m}\right. \\
& +4(p-1) \frac{1}{H^{2}}\left\langle\nabla_{i} f, \nabla_{i} H\right\rangle_{|A|^{2} g-H h}-\frac{2 p-1}{p} \frac{R}{H^{3}}\left(H C-|A|^{4}\right)(5.2) \\
& \left.-\frac{2 R}{H^{3}}\left(|\nabla A|^{2}+2(p-1) \frac{|A|^{2}}{H^{2}}|\nabla H|^{2}-\frac{2 p-1}{H}\left|\nabla_{i} H\right|_{h}^{2}\right)\right\} .
\end{aligned}
$$

Proof. The evolution equation for $f$ is deduced from (4.2) using

$$
\dot{f}_{q}^{p}=-\frac{2}{H^{3}}\left(|A|^{2} \delta_{q}^{p}-H h_{q}^{p}\right)
$$


and

$$
\ddot{f}_{q k}^{p l}=-\frac{2}{H^{3}}\left(h_{k}^{l} \delta_{q}^{p}-H \delta_{k}^{p} \delta_{q}^{l}\right)-\frac{3}{H} \delta_{k}^{l} \dot{f}_{q}^{p}
$$

Hence we have

$$
\begin{aligned}
\frac{\partial f}{\partial t}= & 2 p R^{p-1}\left\{\Delta_{m} f+\frac{2}{H^{3}}\left\langle\nabla_{i} H, A \nabla_{i} A\right\rangle_{m}-\frac{2}{H^{2}}\left|\nabla_{i} A\right|_{m}^{2}\right. \\
& +\frac{3}{H}\left\langle\nabla_{i} f, \nabla_{i} H\right\rangle_{m}-\frac{2}{H^{3}}\left|\nabla_{i} H\right|_{|A|^{2} g-H h}^{2}+\frac{2}{H^{3}}\left|\nabla_{i} A\right|_{|A|^{2} g-H h}^{2} \\
& \left.-(p-1) \frac{1}{R H^{3}}\left|\nabla_{i} R\right|_{|A|^{2} g-H h}^{2}+\frac{2 p-1}{p} \frac{R}{H^{3}}\left(|A|^{4}-H C\right)\right\} .
\end{aligned}
$$

Let us observe that $|A|^{2} g-H h=H(H g-h)-R g$ and that

$$
\nabla R=\nabla\left(\frac{R}{H^{2}} H^{2}\right)=-H^{2} \nabla f+2 \frac{R}{H} \nabla H,
$$

then

$$
\langle\nabla R, \nabla R\rangle=H^{4}\langle\nabla f, \nabla f\rangle-4 R H\langle\nabla f, \nabla H\rangle+4 \frac{R^{2}}{H^{2}}\langle\nabla H, \nabla H\rangle .
$$

The same identity holds with $\langle\cdot, \cdot\rangle_{|A|^{2} g-H h}$. Thus the equation for $f$ becomes

$$
\begin{aligned}
\frac{\partial f}{\partial t}= & 2 p R^{p-1}\left\{\Delta_{m} f+\frac{2}{H^{3}}\left\langle\nabla_{i} H, A \nabla_{i} A\right\rangle_{m}+\frac{3}{H}\left\langle\nabla_{i} f, \nabla_{i} H\right\rangle_{m}\right. \\
& -\frac{2}{H^{3}}\left|\nabla_{i} H\right|_{|A|^{2} g-H h}^{2}-2 \frac{R}{H^{3}}|\nabla A|^{2}-(p-1) \frac{H}{R}\left|\nabla_{i} f\right|_{|A|^{2} g-H h}^{2} \\
& +4(p-1) \frac{1}{H^{2}}\left\langle\nabla_{i} f, \nabla_{i} H\right\rangle_{|A|^{2} g-H h}-4(p-1) \frac{R}{H^{5}}\left|\nabla_{i} H\right|_{|A|^{2} g-H h}^{2} \\
& \left.+\frac{2 p-1}{p} \frac{R}{H^{3}}\left(|A|^{4}-H C\right)\right\} .
\end{aligned}
$$

To obtain equation (5.2) we apply the equality

$$
\begin{aligned}
\frac{2}{H^{3}}\left\langle\nabla_{i} H, A \nabla_{i} A\right\rangle_{m} & =\frac{1}{H}\left\langle\nabla_{i} f, \nabla_{i} H\right\rangle_{m}+2 \frac{|A|^{2}}{H^{4}}\left|\nabla_{i} H\right|_{m}^{2} \\
& =\frac{1}{H}\left\langle\nabla_{i} f, \nabla_{i} H\right\rangle_{m}+2 \frac{R}{H^{4}}\left|\nabla_{i} H\right|_{h}^{2}+\frac{2}{H^{3}}|\nabla H|_{|A|^{2} g-H h}^{2}
\end{aligned}
$$

and we rewrite the quadratic terms in $\nabla H$ as

$$
\begin{aligned}
& -4(p-1) \frac{R}{H^{5}}\left|\nabla_{i} H\right|_{|A|^{2} g-H h}^{2}+2 \frac{R}{H^{4}}|\nabla H|_{h}^{2} \\
& =-2 \frac{R}{H^{3}}\left(2(p-1) \frac{|A|^{2}}{H^{2}}|\nabla H|^{2}-(2 p-1) \frac{1}{H}\left|\nabla_{i} H\right|_{h}^{2}\right) .
\end{aligned}
$$


In the next lemma we estimate the gradient terms in the evolution equation (5.2) not containing $\nabla f$.

Lemma 5.6. Let $f \leq \gamma^{*}(n, p)-\varepsilon$ for some $\varepsilon>0$.

(i) Suppose that either $n>2$ or $p \geq \frac{33}{62}$. Then there exists $\kappa=\kappa(\varepsilon, n, p)>0$ such that

$$
|\nabla A|^{2}+2(p-1) \frac{|A|^{2}}{H^{2}}|\nabla H|^{2}-(2 p-1) \frac{1}{H}\left|\nabla_{i} H\right|_{h}^{2} \geq \kappa|\nabla H|^{2} .
$$

(ii) If $n=2$ and $\frac{1}{2}<p<\frac{33}{62}$ then

$$
|\nabla A|^{2}+2(p-1) \frac{|A|^{2}}{H^{2}}|\nabla H|^{2}-(2 p-1) \frac{1}{H}\left|\nabla_{i} H\right|_{h}^{2} \geq \frac{\varepsilon^{2}}{8}|\nabla H|^{2}+H\langle\nabla f, \nabla H\rangle .
$$

Proof. (i) We recall that, by Lemma 2.2 in [14], $|\nabla A|^{2} \geq 3 /(n+2)|\nabla H|^{2}$. In addition, we have

$$
\left|\nabla_{i} H\right|_{h}^{2}=h^{i j} \nabla_{i} H \nabla_{j} H \leq \lambda_{n}|\nabla H|^{2} .
$$

Therefore, by Proposition 5.1 ii) we have

$$
\begin{aligned}
& |\nabla A|^{2}+2(p-1) \frac{|A|^{2}}{H^{2}}|\nabla H|^{2}-(2 p-1) \frac{1}{H}\left|\nabla_{i} H\right|_{h}^{2} \\
& \geq\left(\frac{3}{n+2}+2(p-1)\left(f+\frac{1}{n}\right)-(2 p-1)\left(\sqrt{\frac{n-1}{n} f}+\frac{1}{n}\right)\right)|\nabla H|^{2} \\
& =\left(\frac{2 n-2}{n(n+2)}+2(p-1) f-(2 p-1) \sqrt{\frac{n-1}{n} f}\right)|\nabla H|^{2}
\end{aligned}
$$

It is convenient to set

$$
\Phi(n, p, f)=\frac{2 n-2}{n(n+2)}+2(p-1) f-(2 p-1) \sqrt{\frac{n-1}{n} f},
$$

where $f$ is considered as an independent variable. We claim that, for any $n \geq 2$ and $p>1 / 2$ (except the case $n=2, p<33 / 62$ ), we have

$$
\Phi(n, p, f)>0 \quad \forall f \in\left[0, \gamma^{*}(n, p)\right) .
$$

Consider first the case $p \geq 1$. Then $2(p-1) f \geq 0$ and we have, by the definition of $\gamma_{2}$,

$$
\Phi(n, p, f) \geq \frac{2 n-2}{n(n+2)}-(2 p-1) \sqrt{\frac{n-1}{n} f}=\frac{2 n-2}{n(n+2)}\left(1-\sqrt{\frac{f}{\gamma_{2}}}\right) .
$$


By definition we have $\gamma^{*} \leq \gamma_{2}$ if $n \geq 4$ and $\gamma^{*}=\gamma_{2}$ if $n=2$, 3. Therefore (5.4) is proved in this case.

The case $1 / 2<p<1$ requires a longer analysis. Let us first show that $\Phi$ is strictly decreasing with respect to $f$ as $f$ varies in $[0,1 / n(n-1)]$. In fact, we have

$$
\begin{aligned}
\frac{\partial \Phi}{\partial f}= & 2(p-1)-\left(p-\frac{1}{2}\right) \sqrt{\frac{n-1}{n f}} \leq 2(p-1)-\left(p-\frac{1}{2}\right)(n-1) \\
= & -\left(p-\frac{1}{2}\right)(n-3)-1 \leq p-\frac{1}{2}-1 \leq-\frac{1}{2}, \\
& \forall f \in\left[0, \frac{1}{n(n-1)}\right] .
\end{aligned}
$$

It follows that, for any $f \in[0,1 / n(n-1))$

$$
\begin{aligned}
\Phi(n, p, f) & >\frac{2 n-2}{n(n+2)}+\frac{2(p-1)}{n(n-1)}-\frac{2 p-1}{n} \\
& =\frac{2 n-2}{n(n+2)}+2(p-1)\left(\frac{1}{n(n-1)}-\frac{1}{n}\right)-\frac{1}{n} \\
& =\frac{n-4}{n(n+2)}+\frac{2(1-p)(n-2)}{n(n-1)}
\end{aligned}
$$

If $n \geq 4$ the above expression is positive. If $n=3$, it is convenient to consider its exact value. We find that

$$
0 \leq f<\frac{1}{n(n-1)} \Longrightarrow \Phi(n, p, f)> \begin{cases}0 & \text { if } n \geq 4 \\ \frac{4-5 p}{15} & \text { if } n=3\end{cases}
$$

By definition, if $n \geq 4$, or if $n=3$ and $p \leq 4 / 5$, then $\gamma^{*}=1 / n(n-1)$. Thus, we have proved (5.4) also in these two cases.

We next consider the case when $n=3, p \in(4 / 5,1)$. Let us observe that, if $0 \leq f \leq 1 / 6$, then by definition of $\Phi$ we have

$$
\begin{aligned}
\Phi(3, p, f) & \geq \frac{4}{15}+2(p-1) \sqrt{\frac{f}{6}}-(2 p-1) \sqrt{\frac{2 f}{3}} \\
& =\frac{4}{15}-p \sqrt{\frac{2 f}{3}}=p \sqrt{\frac{2}{3}}\left(\sqrt{\frac{8}{75 p^{2}}}-\sqrt{f}\right) .
\end{aligned}
$$

Since $8 /\left(75 p^{2}\right)=\gamma^{*}(3, p) \leq 1 / 6$ for all $p \in(4 / 5,1)$, we conclude that $(5.4)$ holds also in this case.

Let us now consider the case $n=2,33 / 62 \leq p<1$. Observe that $(3-$ $2 p) / 8 \leq 15 / 62<1 / n(n-1)$. Therefore, by (5.6), $\Phi$ is decreasing in $f$ for $0<$ 
$f \leq(3-2 p) / 8$, and to prove (5.4) it suffices to check that $\Phi(2, p,(3-2 p) / 8)>0$ for all $p \in[33 / 62,1)$. By the definition of $\Phi$, this means that

$$
1+(p-1)(3-2 p)-(2 p-1) \sqrt{3-2 p} \geq 0, \quad \frac{33}{62} \leq p<1 .
$$

Now, a direct computation shows that the left hand side can be also written as

$$
\left(p-\frac{1}{2}\right)(\sqrt{3-2 p}-1)^{2} \text {. }
$$

Using this expression we immediately see that (5.6) holds. This completes the verification of (5.4).

To conclude the proof of part (i), let us fix any $n, p, \varepsilon$ as in the assertion. By (5.4), the function $f \rightarrow \Phi(n, p, f)$ is positive for $f \in\left[0, \gamma^{*}(n, p)-\varepsilon\right]$. By compactness, it has a minimum $\kappa=\kappa(n, p, \varepsilon)>0$. The lemma follows applying (5.4).

(ii) We consider now the remaining case, where $n=2$ and $1 / 2 \leq p<33 / 62$. Using the identity

$$
|H \nabla A-A \nabla H|^{2}=H^{2}|\nabla A|^{2}-H^{3}\langle\nabla f, \nabla H\rangle-|A|^{2}|\nabla H|^{2},
$$

we rewrite our gradient terms in the form

$$
\begin{aligned}
|\nabla A|^{2}+ & 2(p-1) \frac{|A|^{2}}{H^{2}}|\nabla H|^{2}-(2 p-1) \frac{1}{H}\left|\nabla_{i} H\right|_{h}^{2} \\
= & \frac{1}{H^{2}}\left(|H \nabla A-A \nabla H|^{2}+(2 p-1)\left|\nabla_{i} H\right|_{|A|^{2} g-H h}^{2}\right) \\
& +H\langle\nabla f, \nabla H\rangle .
\end{aligned}
$$

We recall that $[14$, Lemma 2.3$]$ states that

$$
|H \nabla A-A \nabla H|^{2} \geq \frac{1}{2} \lambda_{1}^{2} H^{2}|\nabla H|^{2} .
$$

In addition, Proposition 5.1 (i) for $n=2$ implies

$$
\lambda_{1} \geq \frac{1}{2}\left(\frac{1}{2}-f\right) H \text {. }
$$

Thus we obtain that

$$
\begin{aligned}
|H \nabla A-A \nabla H|^{2} & \geq \frac{1}{8}\left(\frac{1}{2}-f\right)^{2} H^{2}|\nabla H|^{2} \\
& \geq \frac{1}{8}\left(\frac{1}{2}-\gamma^{*}+\varepsilon\right)\left(\frac{1}{2}-f\right) H^{2}|\nabla H|^{2} \\
& =\left(\frac{2 p-1}{2}+\frac{\varepsilon}{8}\right)\left(\frac{1}{2}-f\right) H^{2}|\nabla H|^{2} .
\end{aligned}
$$


On the other hand, when $n=2$ we have

$$
|A|^{2}-\lambda_{n} H=\lambda_{1}^{2}-\lambda_{1} \lambda_{2} \geq-\lambda_{1} \lambda_{2}=-\left(\frac{1}{4}-\frac{f}{2}\right) H^{2} .
$$

Therefore

$$
\left|\nabla_{i} H\right|_{|A|^{2} g-H h}^{2} \geq\left(|A|^{2}-\lambda_{n} H\right)|\nabla H|^{2} \geq-\frac{1}{2}\left(\frac{1}{2}-f\right) H^{2}|\nabla H|^{2} .
$$

We recall that $f \leq \gamma^{*}-\varepsilon<1 / 2-\varepsilon$. Thus, from (5.8) and (5.9) we conclude that

$$
\begin{aligned}
|H \nabla A-A \nabla H|^{2} & +(2 p-1)\left|\nabla_{i} H\right|_{|A|^{2} g-H h}^{2} \\
& \geq \frac{\varepsilon}{8}\left(\frac{1}{2}-f\right) H^{2}|\nabla H|^{2} \geq \frac{\varepsilon^{2}}{8} H^{2}|\nabla H|^{2},
\end{aligned}
$$

which, together with (5.7), yields the conclusion.

The next corollary establishes the monotonicity of $f$.

Corollary 5.7. If $f<\gamma^{*}$ on the initial surface $M_{0}$ of the flow (1.1), then the same holds on $M_{t}$ as long as it exists. Therefore, the solution remains uniformly convex.

Proof. We consider the evolution equation (5.2). If we have $f<\gamma^{*}$ on $M_{0}$, then by compactness we also have $f \leq \gamma^{*}-\varepsilon$ for some $\varepsilon>0$. Then, the previous lemma shows that the gradient terms not containing $\nabla f$ give a negative contribution. Let us analyze the zero order terms. Proposition 5.1 i) implies that $h_{i j} \geq(n-1)(\varepsilon / 2) H g_{i j}$ and we can apply [14, Lemma 2.3] to obtain

$$
H C-|A|^{4} \geq \frac{n(n-1)^{2} \varepsilon^{2}}{4} f H^{4} \geq 0 .
$$

Thus, we can conclude by the maximum principle.

Before proceeding further, it is convenient to define

$$
\varepsilon_{0}=\frac{1}{n(n-1)}-\max _{M_{0}} f>0 .
$$

By the previous result, we have $f \leq 1 / n(n-1)-\varepsilon_{0}$ on $M_{t}$ for any $t>0$. Therefore

$$
R=\left(-f+\frac{n-1}{n}\right) H^{2} \geq\left(\frac{n-2}{n-1}+\varepsilon_{0}\right) H^{2} \geq \varepsilon_{0} H^{2} .
$$

Taking into account that $|A|^{2} \leq H^{2}$ on any convex hypersurface, we conclude that

$$
|A|^{2} \leq H^{2} \leq \frac{R}{\varepsilon_{0}}
$$

which shows that a bound on $R$ implies a bound on all principal curvatures.

We can now prove a stronger invariance result satisfied by our function $f$. 
Theorem 5.8. If we have $f<\gamma^{*}(n, p)$ on $M_{0}$, then there exist two constants $\sigma>0$ and $c_{1}>0$ such that $f \leq c_{1} H^{-\sigma}$ on $M_{t}$, that is

$$
|A|^{2}-\frac{1}{n} H^{2} \leq c_{1} H^{2-\sigma} \quad \forall t \in[0, T) .
$$

Proof. We will study the evolution equation for $f_{\sigma}:=f H^{\sigma}$ with $\sigma>0$ and prove its monotonicity for $\sigma$ small enough. Since we are interested in small values of $\sigma$, it is not restrictive to assume that $\sigma<1$.

The evolution equation for $H^{\sigma}$ can be obtained from (4.2) using that $\dot{H}^{\sigma}=$ $\sigma H^{\sigma-1} I d, \ddot{H}^{\sigma}=\sigma(\sigma-1) H^{\sigma-2} I d \otimes I d$ and applying formula (5):

$$
\begin{aligned}
\frac{\partial H^{\sigma}}{\partial t}= & 2 p R^{p-1}\left\{\Delta_{m} H^{\sigma}+\sigma(1-\sigma) H^{\sigma-2}\left|\nabla_{i} H\right|_{m}^{2}\right. \\
& +\sigma H^{\sigma-1}|\nabla H|^{2}-\sigma H^{\sigma-1}|\nabla A|^{2}+\frac{p-1}{2} \sigma \frac{H^{\sigma+3}}{R}|\nabla f|^{2} \\
& -2(p-1) \sigma H^{\sigma}\langle\nabla f, \nabla H\rangle+2(p-1) \sigma H^{\sigma-1} \frac{R}{H^{2}}|\nabla H|^{2} \\
& \left.-\frac{2 p-1}{2 p} \sigma H^{\sigma-1} R|A|^{2}+\sigma\left(H|A|^{2}-C\right) H^{\sigma}\right\}
\end{aligned}
$$

Let us first estimate from above the terms in the right hand side. Observe that, by definition of $m_{i j}$, we have $\left|\nabla_{i} H\right|_{m}^{2} \leq H|\nabla H|^{2}$. Observe also that $|p-1|<p$ for $p>1 / 2$. In addition, we can use inequality (5.13) and

$$
\left|2 H^{\sigma}\langle\nabla f, \nabla H\rangle\right| \leq H^{\sigma+1}|\nabla f|^{2}+H^{\sigma-1}|\nabla H|^{2}
$$

in order to obtain, after neglecting some negative terms,

$$
\begin{aligned}
\frac{\partial H^{\sigma}}{\partial t} & \leq 2 p R^{p-1}\left\{\Delta_{m} H^{\sigma}+\sigma(2+3 p) H^{\sigma-1}|\nabla H|^{2}\right. \\
& \left.+\left(1+\varepsilon_{0}^{-1}\right) \sigma p H^{\sigma+1}|\nabla f|^{2}+\sigma H^{\sigma+1}|A|^{2}\right\} .
\end{aligned}
$$

We restrict ourselves to the values of $n, p$ covered by case (i) of Lemma 5.6, since the computations in the other case are completely analogous. Then, we obtain from (5.2), (5.15), and Lemma 5.6-(i) that $f_{\sigma}$ satisfies

$$
\begin{aligned}
\frac{\partial f_{\sigma}}{\partial t} \leq & 2 p R^{p-1}\left\{\Delta_{m} f_{\sigma}+(4-2 \sigma) H^{\sigma-1}\left\langle\nabla_{i} f, \nabla_{i} H\right\rangle_{m}\right. \\
& -(p-1) \frac{H^{\sigma+1}}{R}\left|\nabla_{i} f\right|_{|A|^{2} g-H h}^{2}+4(p-1) H^{\sigma-2}\left\langle\nabla_{i} f, \nabla_{i} H\right\rangle_{|A|^{2} g-H h} \\
& -2 \kappa R H^{\sigma-3}|\nabla H|^{2}+7 \sigma p f H^{\sigma-1}|\nabla H|^{2}+\left(1+\varepsilon_{0}^{-1}\right) \sigma p f H^{\sigma+1}|\nabla f|^{2} \\
& \left.-\frac{2 p-1}{p} \frac{R}{H^{3}} H^{\sigma}\left(H C-|A|^{4}\right)+\sigma f H^{\sigma+1}|A|^{2}\right\},
\end{aligned}
$$


where we have also used $2+3 p \leq 7 p$ which follows from $p \geq 1 / 2$. Let us denote by $\mathcal{F}$ the terms involving $\nabla f$, that is,

$$
\begin{aligned}
\mathcal{F}:= & (4-2 \sigma) H^{\sigma-1}\left\langle\nabla_{i} f, \nabla_{i} H\right\rangle_{m}-(p-1) \frac{H^{\sigma+1}}{R}\left|\nabla_{i} f\right|_{|A|^{2} g-H h}^{2} \\
& +4(p-1) H^{\sigma-2}\left\langle\nabla_{i} f, \nabla_{i} H\right\rangle_{|A|^{2} g-H h}+\left(1+\varepsilon_{0}^{-1}\right) \sigma p f H^{\sigma+1}|\nabla f|^{2} .
\end{aligned}
$$

Keeping into account also (5.11), (5.13) and the property that $|A|^{2} \leq H^{2}$ on a convex surface, we can write

$$
\begin{aligned}
\frac{\partial f_{\sigma}}{\partial t} \leq & 2 p R^{p-1}\left\{\Delta_{m} f_{\sigma}+\mathcal{F}-2 \varepsilon_{0} \kappa H^{\sigma-1}|\nabla H|^{2}+7 \sigma p f H^{\sigma-1}|\nabla H|^{2}\right. \\
& \left.-n(n-1)^{2} \varepsilon_{0}^{3} \frac{2 p-1}{4 p} H^{3+\sigma}+\sigma f H^{3+\sigma}\right\} .
\end{aligned}
$$

To apply the maximum principle, it is convenient to write $\mathcal{F}$ in terms of $\nabla f_{\sigma}$, keeping into account that

$$
\nabla f=\nabla\left(f_{\sigma} H^{-\sigma}\right)=H^{-\sigma} \nabla f_{\sigma}-\sigma f H^{-1} \nabla H .
$$

Therefore, denoting by $\mathcal{Z}$ quantities that vanish when $\nabla f_{\sigma}=0$, we obtain

$$
\begin{aligned}
\mathcal{F}= & \mathcal{Z}-\sigma(4-2 \sigma) f H^{\sigma-2}\left|\nabla_{i} H\right|_{m}^{2}-(p-1) \sigma^{2} H^{\sigma-1} \frac{f^{2}}{R}\left|\nabla_{i} H\right|_{|A|^{2} g-H h}^{2} \\
& -4 \sigma(p-1) f H^{\sigma-3}\left|\nabla_{i} H\right|_{|A|^{2} g-H h}^{2}+\left(1+\varepsilon_{0}^{-1}\right) \sigma^{3} p f^{3} H^{\sigma-1}|\nabla H|^{2} \\
\leq & \mathcal{Z}+p \sigma^{2} H^{1+\sigma} \frac{f^{2}}{R}|\nabla H|^{2} \\
& +4 \sigma p f H^{\sigma-1}|\nabla H|^{2}+\left(1+\varepsilon_{0}^{-1}\right) \sigma^{3} p f^{3} H^{\sigma-1}|\nabla H|^{2} \\
\leq & \mathcal{Z}+7 p \sigma \varepsilon_{0}^{-1} H^{\sigma-1}|\nabla H|^{2},
\end{aligned}
$$

where we have used (5.13) and the inequalities $|p-1| \leq p, f<1, \sigma<1, \varepsilon_{0}<1$ and $\left.\left.|| \nabla_{i} H\right|_{|A|^{2} g-H h} ^{2}\left|\leq H^{2}\right| \nabla H\right|^{2}$. Thus

$$
\begin{aligned}
\frac{\partial f_{\sigma}}{\partial t} \leq & 2 p R^{p-1}\left\{\Delta_{m} f_{\sigma}+\mathcal{Z}-2 \varepsilon_{0} \kappa H^{\sigma-1}|\nabla H|^{2}+7\left(1+\varepsilon_{0}^{-1}\right) \sigma p H^{\sigma-1}|\nabla H|^{2}\right. \\
& \left.-n(n-1)^{2} \varepsilon_{0}^{3} \frac{2 p-1}{4 p} H^{3+\sigma}+\sigma f H^{3+\sigma}\right\} .
\end{aligned}
$$

Now if we choose $\sigma>0$ small enough to have

$$
7\left(1+\varepsilon_{0}^{-1}\right) \sigma p \leq 2 \varepsilon_{0} \kappa, \quad \sigma \leq n(n-1)^{2} \varepsilon_{0}^{3} \frac{2 p-1}{4 p},
$$

we can apply the maximum principle to conclude that $f_{\sigma}$ is nondecreasing, hence $f \leq c_{1} H^{-\sigma}$. 


\section{Convergence to a point}

In this section we prove the first part of Theorem 1.1. We largely follow the method of Tso [23] and Andrews [2], based on the analysis of the behaviour of the inner and outer radius of the surface (see the next definition). We show, on one hand, that the inner radius tends to zero at the singular time; on the other hand, as a consequence of the pinching estimate, the outer radius is comparable to the inner radius and also tends to zero, implying the convergence to a point.

Definition 6.1. We define the inner and outer radius of $M_{t}$ respectively as:

$$
\begin{aligned}
& \rho_{-}(t)=\sup \left\{r \geq 0 \mid B_{r}(\mathbf{y}) \text { is enclosed by } \mathbf{F}_{t}(M) \text { for some } \mathbf{y} \in \mathbb{R}^{n+1}\right\} \\
& \rho_{+}(t)=\inf \left\{r \geq 0 \mid B_{r}(\mathbf{y}) \text { encloses } \mathbf{F}_{t}(M) \text { for some } \mathbf{y} \in \mathbb{R}^{n+1}\right\}
\end{aligned}
$$

where $B_{r}(\mathbf{y})$ is the ball of radius $r$ centered at $\mathbf{y}$.

Let us observe that, since the speed of the flow is always directed towards the interior of the surface, any ball which encloses (rispectively is enclosed by) $\mathbf{F}_{t_{0}}(M)$ at some time $t_{0}$ also encloses (rispectively is enclosed by) $\mathbf{F}_{t}(M)$ for $t>t_{0}$ (rispectively $\left.t<t_{0}\right)$. It follows that $\rho_{-}(t)$ and $\rho_{+}(t)$ are decreasing functions of $t$. It was observed by Andrews [2], that the pinching of the curvatures implies a bound on the ratio $\rho_{+} / \rho_{-}$, as stated in the next lemma. In the following, we denote by $c_{i}$, $i \geq 2$ constants depending on $n, p$ and on the initial surface, but independent on $t$ (like the constant $c_{1}$ in the statement of Theorem 5.8).

Lemma 6.2. Let $M_{t}$ be a solution of the flow (1.1). If the initial surface satisfies the hypotheses of Theorem 5.8, then there exists $c_{2}>0$ such that

$$
\rho_{+}(t) \leq c_{2} \rho_{-}(t) \quad \forall t \in[0, T) .
$$

Proof. Thanks to Corollary 5.7, we have $f \leq 1 / n(n-1)-\varepsilon_{0}$ for all times $t \in$ $[0, T)$, where $\varepsilon_{0}$ is given by (5.12). Using Proposition 5.1 (i), we have on $M_{t}$

$$
\lambda_{1} \geq \frac{(n-1) \varepsilon_{0}}{2} H \geq \frac{(n-1) \varepsilon_{0}}{2} \lambda_{n} .
$$

This allows us to apply Theorem 5.1 and Theorem 5.4 of [2] to prove the statement.

We now prove that the principal curvatures of the evolving surfaces stay bounded as long as the inner radius is positive. As in $[2,23]$, we derive this result by studying the properties of the function

$$
v:=\frac{R^{p}}{2\langle\mathbf{F}, v\rangle-r}
$$


for some $r>0$. It can be checked by a straightforward computation that, if $\mathbf{F}$ is a solution of our flow, then $v$ satisfies

$$
\begin{aligned}
\frac{\partial v}{\partial t}= & 2 p R^{p-1}\left(\Delta_{m} v+\frac{4\left\langle\nabla_{i}\langle\mathbf{F}, v\rangle, \nabla_{i} v\right\rangle_{m}}{2\langle\mathbf{F}, v\rangle-r}\right) \\
& +2(2 p+1) v^{2}-\frac{r\left(H^{2}|A|^{2}-C\right) v^{2}}{R} .
\end{aligned}
$$

Proposition 6.3. Given any $T^{\prime} \in(0, T)$, we have

$$
\max _{M_{t}} R \leq c_{4}\left(1+\frac{1}{\rho_{-}\left(T^{\prime}\right)^{2}}\right) \quad \forall t \in\left[0, T^{\prime}\right]
$$

for some $c_{4}>0$ (independent of $r, T^{\prime}$ ).

Proof. Let us take $\left(\mathbf{p}^{*}, t^{*}\right)$ such that $\max _{M \times\left[0, T^{\prime}\right]} R=R\left(\mathbf{p}^{*}, t^{*}\right)$. If we set $r=\rho_{-}\left(t^{*}\right)$, there exists $\mathbf{y}_{0} \in \mathbb{R}^{n+1}$ such that the sphere $B_{r}\left(\mathbf{y}_{0}\right)$ is enclosed in $M_{t}$ for any $t \in\left[0, t^{*}\right]$. Now we call $\mathbf{Y}:=\mathbf{F}-\mathbf{y}_{0}$ the position vector field with origin $\mathbf{y}_{0}$ and we note that

$$
2\langle\mathbf{Y}, v\rangle-r \geq r>0
$$

on $M_{t}$ for any $t \in\left[0, t^{*}\right]$, as a consequence of the convexity of $M_{t}$. Thus the quantity $v:=R^{p} /(2\langle\mathbf{Y}, v\rangle-r)$ is well defined on $M \times\left[0, t^{*}\right]$, and satisfies the evolution equation (6.2) with $\mathbf{F}$ replaced by $\mathbf{Y}$. In order to apply the maximum principle we use formula (4.4) to estimate

$$
\frac{\partial v}{\partial t} \leq 2 p R^{p-1}\left(\Delta_{m} v+\frac{4\left\langle\nabla_{i}\langle\mathbf{Y}, v\rangle, \nabla_{i} v\right\rangle_{m}}{2\langle\mathbf{Y}, v\rangle-r}\right)+2(2 p+1)\left[1-\frac{r}{c_{3}} R^{\frac{1}{2}}\right] v^{2},
$$

where we have set

$$
c_{3}:=\frac{2 p+1}{p} \sqrt{n-1}(\sqrt{n}+1) .
$$

Assume that $v$ attains a local maximum at $\left(\mathbf{p}_{0}, t_{0}\right)$ with $t_{0}>0$. Then at $\left(\mathbf{p}_{0}, t_{0}\right)$ we have $\Delta_{m} v \leq 0, \nabla v=0$ and $\frac{\partial v}{\partial t} \geq 0$. Thus,

$$
R^{\frac{1}{2}}\left(p_{0}, t_{0}\right) \leq \frac{c_{3}}{r}
$$

We deduce that

$$
\max _{M \times\left[0, t^{*}\right]} v \leq \max \left\{\max _{M_{0}} v(\cdot, 0), \frac{c_{3}^{2 p}}{r^{2 p+1}}\right\} .
$$

Let us first consider the case where $\max v(\cdot, 0) \leq c_{3}^{2 p} / r^{2 p+1}$. Since we have $\left|\mathbf{F}\left(\mathbf{p}^{*}, t^{*}\right)-\mathbf{y}_{0}\right| \leq \operatorname{diam}\left(M_{t^{*}}\right) \leq 2 \rho_{+}\left(t^{*}\right)$, we obtain, recalling (6.1),

$$
\begin{aligned}
R^{p}\left(\mathbf{p}^{*}, t^{*}\right) & =v\left(\mathbf{p}^{*}, t^{*}\right)\left(2\left\langle\mathbf{F}\left(\mathbf{p}^{*}, t^{*}\right)-\mathbf{y}_{0}, v\right\rangle-r\right) \leq \frac{c_{3}^{2 p}}{r^{2 p+1}}\left(4 \rho_{+}\left(t^{*}\right)-r\right) \\
& \leq \frac{c_{3}^{2 p}}{r^{2 p+1}}\left(4 c_{2} \rho_{-}\left(t^{*}\right)-r\right)=\frac{c_{3}^{2 p}}{r^{2 p}}\left(4 c_{2}-1\right)
\end{aligned}
$$


If we have instead $\max v(\cdot, 0)>c_{3}^{2 p} / r^{2 p+1}$, we observe that

$$
\max _{M_{0}} v(\cdot, 0) \leq \frac{1}{r} \max _{M_{0}} R^{p} .
$$

Then, similarly as before, we find

$$
R^{p}\left(\mathbf{p}^{*}, t^{*}\right)=\left(\frac{1}{r} \max _{M_{0}} R^{p}\right)\left(4 \rho_{+}\left(t^{*}\right)-r\right) \leq\left(\max _{M_{0}} R^{p}\right)\left(4 c_{2}-1\right) .
$$

Since $r=\rho_{-}\left(t^{*}\right) \leq \rho_{-}\left(T^{\prime}\right)$, the assertion follows from (6.4) and (6.5).

Now we can apply the regularity result from Section 3 to prove that the evolving surfaces converge to a point as they approach the singular time.

Theorem 6.4. The solution to the problem (1.1) exists in a finite time interval $[0, T)$ and the $M_{t}$ 's converge to a point as $t \rightarrow T$.

Proof. We know from Corollary 4.3 that the maximal existence time $T$ of the solution is finite and that $|A|^{2}$ becomes unbounded as $t \rightarrow T$. By estimate (5.13), the scalar curvature $R$ also becomes unbounded as $t \rightarrow T$. We deduce from Proposition 6.3 that $\rho_{-}(t)$, which is a decreasing quantity, tends to zero as $t \rightarrow T$. Finally, inequality (6.1) implies that also the outer radius tends to zero. Hence, the evolving surfaces shrink to a point.

\section{Convergence to a sphere}

In order to prove the second part of Theorem 1.1, we consider the rescaled maps $\widetilde{\mathbf{F}}(\mathbf{p}, t)=\psi(t)\left(\mathbf{F}(\mathbf{p}, t)-\mathbf{y}_{T}\right)$ where $\mathbf{y}_{T}$ is the point to which $M_{t}$ converges as $t \rightarrow T$ and

$$
\psi(t)=\left[c^{\prime}(T-t)\right]^{-\frac{1}{2 p+1}},
$$

where $c^{\prime}=n^{p}(n-1)^{p}(2 p+1)$. The factor $\psi$ is chosen in such a way that a sphere shrinking to $\mathbf{y}_{T}$ as $t \rightarrow T$ in the original flow becomes a sphere with constant radius one after rescaling. In addition, we introduce a new time parameter $\tau$, defined as

$$
\tau(t):=\int_{0}^{t} \frac{1}{c^{\prime}\left(T-t^{\prime}\right)} d t^{\prime}=-\frac{1}{c^{\prime}} \ln \left(1-\frac{t}{T}\right) .
$$

Then $\tau \rightarrow+\infty$ as $t \rightarrow T$.

Let us denote with a tilde the geometric quantities associated with the rescaled $R^{p}$-flow. A straightforward computation yields:

Lemma 7.1. The rescaled immersions satisfy the equation

$$
\frac{\partial}{\partial \tau} \widetilde{\mathbf{F}}=-\widetilde{R}^{p} \tilde{v}+n^{p}(n-1)^{p} \widetilde{\mathbf{F}} .
$$


The associated volume element $d \tilde{\mu}=\psi^{n} d \mu$ evolves according to

$$
\frac{\partial(d \tilde{\mu})}{\partial \tau}=-\widetilde{R}^{p} \widetilde{H} d \tilde{\mu}+n^{p+1}(n-1)^{p} d \tilde{\mu} .
$$

In general, if $P$ is a homogeneous function of degree $\alpha$ in the curvatures satisfying the equation

$$
\frac{\partial P}{\partial t}=2 p R^{p-1}\left(\Delta_{m} P+Q\right),
$$

for some function $Q$, then the rescaled quantity $\widetilde{P}=\psi^{-\alpha} P$ satisfies

$$
\frac{\partial \widetilde{P}}{\partial \tau}=2 p \widetilde{R}^{p-1}\left(\Delta_{\tilde{m}} \widetilde{P}+\widetilde{Q}\right)-\alpha n^{p}(n-1)^{p} \widetilde{P}
$$

where $\widetilde{Q}=\psi^{-\alpha-3} Q$.

In this section we denote with $c_{i}^{\prime}, i \geq 1$, constants associated with the rescaled flow which are independent of $\tau$. Following the proof of [2, Theorem 7.1], we can prove the estimate

$$
\frac{1}{c_{1}^{\prime}} \leq \tilde{\rho}_{-}(\tau) \leq 1 \leq \tilde{\rho}_{+}(\tau) \leq c_{1}^{\prime}, \quad \tau \in[0,+\infty) .
$$

This implies in particular that the area of the surfaces $\widetilde{M}_{\tau}:=\widetilde{\mathbf{F}}(\cdot, \tau)$, by convexity, is uniformly bounded both from above and from below. Using Proposition 6.3 we can also estimate from above the rescaled scalar curvature

$$
\max _{\widetilde{M}_{\tau}} \widetilde{R}=\psi^{-2} \max _{M_{t}} R=c_{4}\left[\psi^{-2}+\frac{1}{\tilde{\rho}_{-}(\tau)^{2}}\right] \leq c_{4}\left[\psi(0)^{-2}+\left(c_{1}^{\prime}\right)^{2}\right] .
$$

Taking into account (5.13), we deduce that on the rescaled surfaces all curvatures are bounded:

$$
|\widetilde{A}|^{2} \leq c_{2}^{\prime}, \quad \widetilde{H}^{2} \leq c_{2}^{\prime}, \quad \widetilde{R} \leq c_{2}^{\prime} .
$$

On the other hand, we do not have an estimate showing that the rescaled scalar curvature is uniformly positive; thus, the principal curvatures of our hypersurface may a priori become arbitrarily small for large values of $\tau$. Observe that the operator $\widetilde{R}^{p-1} \Delta_{\tilde{m}}$, which appears in the equations of the form (7.3), has coefficients which are homogeneous of degree $2 p-1$ in the curvatures. Therefore, we cannot ensure that the coefficients are bounded away from zero, and the corresponding equations should be treated as degenerate parabolic. Following the procedure of Schulze [22], we will derive a Hölder estimate on the scalar curvature using a result due to Di Benedetto and Friedman [11, Theorem 1.3] for solutions of equations of porous medium type in domains of the euclidean space. Roughly speaking, the result in [11] is the following: let $v(x, t)$ be a nonnegative solution of

$$
\frac{\partial v}{\partial t}-D_{i}\left(a^{i j}(x, t, D v) D_{j} v^{m}\right)=f(x, t, v, D v), \quad(x, t) \in B_{r} \times[0, T],
$$


where $m>1$ and the coefficients $a^{i j}$ are uniformly elliptic. Suppose in addition that $v$ satisfies

$$
\begin{aligned}
& |f(x, t, v, D v)| \leq k_{1}\left|D v^{m}\right|+k_{2}, \\
& \sup _{0<t<T}|| v(\cdot, t)\left\|_{L^{2}\left(B_{r}\right)}^{2}+\right\| D v^{m} \|_{L^{2}\left(B_{r} \times[0, T]\right)}^{2} \leq k_{3}
\end{aligned}
$$

for suitable constants $k_{1}, k_{2}, k_{3}$. Then $v$ satisfies an interior $C^{\alpha}$ estimate with a constant depending only on the ellipticity of the $a^{i j}$ and on the constants $k_{i}$.

The application of this result to our problem requires some additional work compared to [22], mainly because of the presence of the operator $\Delta_{m}$ rather then $\Delta$ in the evolution equations. Let us first derive an integral bound on $\left|\nabla \widetilde{R}^{p}\right|$ which will be used later in the verification of (7.9).

Lemma 7.2. For any $\tau_{2}>\tau_{1}>0$, the rescaled scalar curvature $\widetilde{R}$ of the evolving surfaces $\tilde{M}_{\tau}$ satisfies the estimate

$$
\int_{\tau_{1}}^{\tau_{2}} \int_{\tilde{M}_{\tau}}\left|\nabla \widetilde{R}^{p}\right|_{\widetilde{m}}^{2} d \tilde{\mu} d \tau \leq c_{4}^{\prime}\left(1+\tau_{2}-\tau_{1}\right)
$$

for a constant $c_{4}^{\prime}$ independent of $\tau_{1}, \tau_{2}$.

Proof. From (4.3) and (7.3) we deduce that

$$
\frac{\partial \widetilde{R}}{\partial \tau}=2 p \widetilde{R}^{p-1}\left\{\Delta_{\tilde{m}} \widetilde{R}+(p-1) \frac{1}{\widetilde{R}}\left|\nabla_{i} \widetilde{R}\right|_{\tilde{m}}^{2}+\frac{1}{p}\left(\widetilde{H}|\widetilde{A}|^{2}-\widetilde{C}\right) \widetilde{R}\right\}-2 n^{p}(n-1)^{p} \widetilde{R}
$$

Using the identity

$$
\Delta_{\tilde{m}} \widetilde{R}^{p}=p(p-1) \widetilde{R}^{p-2}\left|\nabla_{i} \widetilde{R}\right|_{\tilde{m}}^{2}+p \widetilde{R}^{p-1} \Delta_{\tilde{m}} \widetilde{R}
$$

the above equation can be written as a porous medium equation:

$$
\frac{\partial}{\partial \tau} \widetilde{R}=2 \Delta_{\tilde{m}} \widetilde{R}^{p}+2\left(\widetilde{H}|\widetilde{A}|^{2}-\widetilde{C}\right) \widetilde{R}^{p}-2 n^{p}(n-1)^{p} \widetilde{R}
$$

Now we observe that the tensor $m_{i j}$ is divergence-free, as a consequence of the Codazzi equations:

$$
\nabla^{i} m_{i j}=\nabla^{i} H g_{i j}-\nabla^{j} h_{i i}=\nabla_{j} H-\nabla_{j} H=0 .
$$

Therefore the operator $\Delta_{m}$ satisfies the following identity, for any two functions $a, b \in C^{2}(M)$ :

$$
\int_{M} a \Delta_{m} b d \mu=-\int_{M}\langle\nabla a, \nabla b\rangle_{m} d \mu
$$


The same properties hold for the rescaled tensor $\tilde{m}$. Hence we have, taking into account also (7.2),

$$
\begin{aligned}
\frac{d}{d \tau} \int \widetilde{R}^{p+1} d \tilde{\mu}= & (p+1) \int \widetilde{R}^{p} \frac{\partial}{\partial \tau} \widetilde{R} d \tilde{\mu}+\int \widetilde{R}^{p+1} \frac{\partial}{\partial \tau} d \tilde{\mu} \\
= & 2(p+1) \int \widetilde{R}^{p} \Delta_{\tilde{m}} \widetilde{R}^{p} d \tilde{\mu}+2(p+1) \int\left(\widetilde{H}|\widetilde{A}|^{2}-\widetilde{C}\right) \widetilde{R}^{2 p} d \tilde{\mu} \\
& -2(p+1) n^{p}(n-1)^{p} \int \widetilde{R}^{p+1} d \tilde{\mu}-\int \widetilde{R}^{2 p+1} \widetilde{H} d \tilde{\mu} \\
& +n^{p+1}(n-1)^{p} \int \widetilde{R}^{p+1} d \tilde{\mu}=-2(p+1) \int\left|\nabla \widetilde{R}^{p}\right|_{\tilde{m}}^{2} d \tilde{\mu} \\
& +2(p+1) \int\left(\widetilde{H}|\widetilde{A}|^{2}-\widetilde{C}\right) \widetilde{R}^{2 p} d \tilde{\mu}-\int \widetilde{R}^{2 p+1} \widetilde{H} d \tilde{\mu} \\
& +n^{p}(n-1)^{p}(n-2 p-2) \int \widetilde{R}^{p+1} d \tilde{\mu} .
\end{aligned}
$$

Now, estimate (7.6) shows that $\widetilde{R}, \widetilde{H},|\widetilde{A}|^{2}$ are all uniformly bounded. It follows that

$$
\int\left|\nabla \widetilde{R}^{p}\right|_{\tilde{m}}^{2} d \tilde{\mu} \leq-\frac{1}{2(p+1)} \frac{d}{d \tau} \int \widetilde{R}^{p+1} d \tilde{\mu}+\int c_{3}^{\prime} d \tilde{\mu} .
$$

Since the area of $\widetilde{M}_{\tau}$ is also bounded, the statement follows integrating in time.

We are now ready to complete the proof of our main Theorem 1.1. We will explain in detail the steps of the procedure which are different from [22], omitting the parts which are analogous.

Theorem 7.3. The rescaled hypersurfaces $\tilde{M}_{\tau}$ converge to a sphere in $C^{\infty}$ norm with exponential speed as $\tau \rightarrow \infty$.

Proof. The crucial step of the proof consists of deriving a uniform Hölder estimate for $\widetilde{R}$. To this purpose, we write locally $\widetilde{M}_{t}$ as a graph, as in the proof of Theorem 3.3. We consider the equation (7.10) satisfied by $\widetilde{R}$ in the local coordinates of the graph representation. If we denote by $D_{i}$ the derivatives with respect to the coordinates, we can write the second order operator in divergence form

$$
\begin{aligned}
\Delta_{\tilde{m}} \widetilde{R}^{p} & =\tilde{m}^{i j}\left(D_{i} D_{j} \widetilde{R}^{p}-\widetilde{\Gamma}_{i j}^{l} D_{l} \widetilde{R}^{p}\right) \\
& =D_{i}\left(\tilde{m}^{i j} D_{j} \widetilde{R}^{p}\right)-D_{i} \tilde{m}^{i j} D_{j} \widetilde{R}^{p}-\tilde{m}^{i j} \widetilde{\Gamma}_{i j}^{l} D_{l} \widetilde{R}^{p}
\end{aligned}
$$

However, this expression is not yet satisfactory: in fact, the coefficients $\tilde{m}^{i j}$ are functions of degree one of the curvatures and therefore tend to zero if if $\widetilde{R}$ tends to zero. Thus, we cannot ensure the uniform ellipticity of the $\tilde{m}^{i j}$ 's. For this reason, 
we rewrite the operator as

$$
\begin{aligned}
\Delta_{\tilde{m}} \widetilde{R}^{p}= & \frac{2 p}{2 p+1} D_{i}\left(\widetilde{R}^{-\frac{1}{2}} \tilde{m}^{i j} D_{j} \widetilde{R}^{p+\frac{1}{2}}\right) \\
& -\frac{2 p}{2 p+1} \widetilde{R}^{-\frac{1}{2}} D_{i} \tilde{m}^{i j} D_{j} \widetilde{R}^{p+\frac{1}{2}}-\tilde{m}^{i j} \widetilde{\Gamma}_{i j}^{l} D_{l} \widetilde{R}^{p} .
\end{aligned}
$$

Now the coefficients $\widetilde{R}^{-\frac{1}{2}} \tilde{m}^{i j}$ are both bounded and uniformly elliptic. In fact, by definition of $m^{i j}$, we have

$$
\lambda_{1} g^{i j} \leq\left(H-\lambda_{n}\right) g^{i j} \leq m^{i j} \leq\left(H-\lambda_{1}\right) g^{i j} \leq H g^{i j} .
$$

Taking into account Proposition 5.1-(i) and (5.13), we see that $\tilde{R}^{-\frac{1}{2}} \tilde{m}^{i j}$ is comparable to $\tilde{g}^{i j}$, which in turn is comparable to the identity matrix in our local graph representation. In this way we can rewrite (7.10) as

$$
\begin{aligned}
\frac{\partial}{\partial \tau} \widetilde{R} & -\frac{4 p}{2 p+1} D_{i}\left(\widetilde{R}^{-\frac{1}{2}} \tilde{m}^{i j} D_{j} \widetilde{R}^{p+\frac{1}{2}}\right) \\
& =-\frac{4 p}{2 p+1} \widetilde{R}^{-\frac{1}{2}} D_{i} \tilde{m}^{i j} D_{j} \widetilde{R}^{p+\frac{1}{2}}-2 \tilde{m}^{i j} \tilde{\Gamma}_{i j}^{l} D_{l} \widetilde{R}^{p}+Q,
\end{aligned}
$$

with $Q$ uniformly bounded by (7.6). Such an equation is of the type (7.7) with $m=p+\frac{1}{2}$. Let us check properties (7.8) and (7.9). By property (7.11), we have

$$
D_{i} \tilde{m}^{i j}=\nabla_{i} \tilde{m}^{i j}-\widetilde{\Gamma}_{i l}^{j} \tilde{m}^{i l}-\widetilde{\Gamma}_{i l}^{i} \tilde{m}^{j l}=-\widetilde{\Gamma}_{i l}^{j} \tilde{m}^{i l}-\widetilde{\Gamma}_{i l}^{i} \tilde{m}^{j l}
$$

Since the Christoffel symbols are uniformly bounded in the local graph representation, we have that $D_{i} \tilde{m}^{i j} \approx \tilde{m}^{i j} \approx \widetilde{R}^{1 / 2}$, and we obtain an estimate on the right hand side of (7.12) in terms of $D \widetilde{R}^{p+\frac{1}{2}}$, as required in (7.8). On the other hand, Lemma 7.2 implies

$$
\int_{\tau_{0}-\varepsilon}^{\tau_{0}+\varepsilon} \int_{B_{\varepsilon}\left(\mathbf{p}_{0}\right) \cap T_{\mathbf{p}_{0}} \tilde{M}_{\tau_{0}}}\left|D \widetilde{R}^{p+\frac{1}{2}}\right|^{2} \leq c_{5}^{\prime} \int_{\tau_{0}-\varepsilon}^{\tau_{0}+\varepsilon} \int_{\tilde{M}_{\tau}}\left|\nabla \widetilde{R}^{p}\right|_{\tilde{m}}^{2} \widetilde{R}^{\frac{1}{2}} \leq c_{6}^{\prime} .
$$

Keeping into account also (7.6), we see that condition (7.9) is satisfied. Thus, Theorem 1.2 of [11] gives the desired $C^{\alpha}$ uniform interior estimate on $\widetilde{R}$.

The rest of the proof can be now done by the same arguments as in [22, Lemma 3.4 and Theorem 3.5]. We just sketch some ideas of the procedure to explain the role of the Hölder estimate proved above, and also of Theorem 5.8, which has not been used yet. First we notice that the upper bound on $\tilde{\rho}_{+}$in (7.4) implies that at any time $\tau$ there is some point where the curvature of $\widetilde{M}_{\tau}$ is greater than some fixed positive constant. The Hölder estimate for $\widetilde{R}$ then shows that there is a whole neighbourhood where the curvature is uniformly positive. At any point where the rescaled curvature remains uniformly positive as $\tau \rightarrow+\infty$, the unrescaled curvature becomes unbounded: then Theorem 5.8 implies that $f$ tends to zero, so that in 
the limit the point is umbilical. These arguments easily imply the existence of a sequence $\tau_{j} \rightarrow+\infty$ such that suitable parts of $\widetilde{M}_{\tau_{j}}$ converge to a portion of a sphere. The above procedure can then be repeated in a larger neighbourhood, so that after finitely many iterations one proves the convergence to a sphere of the whole surfaces $\widetilde{M}_{\tau_{j}}$. Standard techniques then allow to prove that the whole flow $\widetilde{M}_{\tau}$ converges to a sphere, and to show that the speed is exponential. We refer to [22] for the details.

\section{Neckpinch singularities}

In this last section we show that the conclusions of Theorem 1.1 are false in dimension $n>2$ if one considers arbitrary nonconvex initial surfaces with positive scalar curvature. As in the case of mean curvature flow, we prove that there are suitable surfaces with the shape of dumbbell which develop a neckpinch singularity in finite time under the scalar curvature flow, and thus do not shrink to a point.

In the counterexample we are going to construct we will use a suitable family of rotationally symmetric surfaces as a barrier; to this purpose it is useful to estimate the norm $|\cdot|_{m}$ as shown by the next lemma.

Lemma 8.1. Let $M \subset \mathbb{R}^{n+1}$ be a hypersurface with $H>0$ and $R>0$. If $M$ is rotationally symmetric with $\lambda_{2}=\ldots=\lambda_{n}$, then at any point we have $\lambda_{n}<\frac{2}{n} H$ and the norm associated to the metric $m_{i j}=H g_{i j}-h_{i j}$ can be estimated by the standard norm $|\cdot|_{g}$ :

$$
\frac{n-2}{n} H|\cdot|_{g}<|\cdot|_{m}<\frac{2(n-1)}{n} H|\cdot|_{g} .
$$

Proof. At any point of the surface $M$, the eigenvalues of the matrix $m_{j}^{i}$ are $H-\lambda_{i}$ for $i=1, \ldots, n$. We recall that, under the hypothesis $H>0$ and $R>0$, Lemma 2.4 in [17] implies $H-\lambda_{n}>0$; then we can set $H-\lambda_{n}=\varepsilon H$, i.e. $\lambda_{n}=(1-\varepsilon) H$, with $0<\varepsilon<1$.

In addition, $\lambda_{1}$ can be expressed as $\lambda_{1}=\varepsilon H-(n-2) \lambda_{n}$ and the scalar curvature $R$ becomes

$$
\begin{aligned}
R & =\lambda_{1}\left(H-\lambda_{1}\right)+(n-1) \lambda_{n}\left(H-\lambda_{n}\right) \\
& =\left[\varepsilon H-(n-2) \lambda_{n}\right](n-1) \lambda_{n}+(n-1) \lambda_{n} \varepsilon H \\
& =(n-1)(1-\varepsilon)[n \varepsilon-(n-2)] H^{2} .
\end{aligned}
$$

Since $\varepsilon<1$, we deduce that $\frac{n-2}{n}<\varepsilon$. Hence

$$
H-\lambda_{n}=\varepsilon H>\frac{n-2}{n} H, \quad \lambda_{n}<\frac{2}{n} H
$$

and

$$
H-\lambda_{1}=(n-1)(1-\varepsilon) H<\frac{2(n-1)}{n} H,
$$

which proves the assertion. 
The example we are going to define is inspired by the construction of Ecker [12] for the mean curvature flow. We first introduce a suitable family $B_{t}$ of hyperboloids which are an outer barrier for our flow in the sense given by the following proposition. We denote by $\mathbf{y}=\left(y_{1}, \ldots, y_{n+1}\right)$ the points of the ambient space $\mathbb{R}^{n+1}$.

Proposition 8.2. Let $M_{0}$ be a closed hypersurface of dimension $n>2$ with positive scalar curvature everywhere, rotationally symmetric with respect to the $y_{n+1}$-axis, and let $M_{t}$, with $t \in[0, T)$, be its evolution under the flow (1.1) for some $p \geq 1 / 2$. Suppose that $M_{0}$ is contained in the strip $-N \leq y_{n+1} \leq N$, for some $N>0$, and that it is enclosed by the hyperboloid $B_{0}$ of equation

$$
2|\mathbf{y}|^{2}-(n-\beta) y_{n+1}^{2}-2 \eta^{2}=0,
$$

where $0<\beta<n-2$ and $0<\eta<1$. Then there exists $k_{0}=k_{0}(N, \beta)$ such that $M_{t}$ is enclosed in the hyperboloid $B_{t}$ of equation

$$
2|\mathbf{y}|^{2}-(n-\beta) y_{n+1}^{2}+2 k_{0} t-2 \eta^{2}=0,
$$

for any $0 \leq t \leq \min \left\{T, \eta^{2} / k_{0}\right\}$. If we assume in addition that $M_{0}$ is symmetric with respect to the hyperplane $y_{n+1}=0$, it follows that its singular time $T$ satisfies $T \leq \eta^{2} / k_{0}$.

Proof. Let us consider the hyperboloids $B_{t}$ as in the above statement for a general $k_{0}>0$. The equation of $B_{t}$ can be written as

$$
2|\hat{\mathbf{y}}|^{2}=(n-2-\beta) y_{n+1}^{2}+2 \eta^{2}-2 k_{0} t
$$

where $\mathbf{y}=\left(\hat{\mathbf{y}}, y_{n+1}\right)$ and $B_{t}$ can also be regarded as the rotation around the $y_{n+1}$ axis of the graph of the function

$$
s_{H}\left(y_{n+1}\right):=\sqrt{\frac{n-2-\beta}{2} y_{n+1}^{2}+\eta^{2}-k_{0} t} .
$$

The principal curvatures of a rotationally symmetric surface are

$$
\lambda_{1}=\frac{-s_{H}^{\prime \prime}}{\left[1+\left(s_{H}^{\prime}\right)^{2}\right]^{\frac{3}{2}}}, \quad \lambda_{k}=\frac{1}{s_{H}\left[1+\left(s_{H}^{\prime}\right)^{2}\right]^{\frac{1}{2}}} k=2, \ldots, n .
$$

Hence the principal curvatures of $B_{t}$ are

$$
\lambda_{1}=-\frac{(n-2-\beta) 4\left(\eta^{2}-k_{0} t\right)}{\left[(n-2-\beta)(n-\beta) y_{n+1}^{2}+4\left(\eta^{2}-k_{0} t\right)\right]^{\frac{3}{2}}}
$$


and

$$
\lambda_{n}=\frac{2}{\left[(n-2-\beta)(n-\beta) y_{n+1}^{2}+4\left(\eta^{2}-k_{0} t\right)\right]^{\frac{1}{2}}} .
$$

Note that $\frac{\lambda_{1}}{\lambda_{n}} \geq-\frac{n-2-\beta}{2}$. This implies that $H=\lambda_{1}+(n-1) \lambda_{n}>0$ on $B_{t}$. In addition, since the scalar curvature is given by

$$
R=(n-1) \lambda_{n}\left[2 \lambda_{1}+(n-2) \lambda_{n}\right]
$$

we have

$$
R \geq(n-1) \beta \lambda_{n}^{2}=\frac{4(n-1) \beta}{(n-2-\beta)(n-\beta) y_{n+1}^{2}+4\left(\eta^{2}-k_{0} t\right)} .
$$

Since we assume $0<\eta<1$, it follows that on any point of $B_{t}$ such that $y_{n+1} \in$ $[-N, N]$ we have

$$
R \geq \frac{4(n-1) \beta}{(n-2-\beta)(n-\beta) N^{2}+4}=: c_{0}(\beta, N) .
$$

Now let us consider the function

$$
h=2|\mathbf{y}|^{2}-(n-\beta) y_{n+1}^{2}+2 k_{0} t-2 \eta^{2}
$$

vanishing on $B_{t}$ and negative inside. An easy computation shows that on the evolving surface $M_{t}$ the function $h$ satisfies

$$
\left(\frac{\partial}{\partial t}-R^{p-1} \Delta_{m}\right) h=2 k_{0}-4(n-1) H R^{p-1}+2(n-\beta) R^{p-1}\left|\nabla_{i} y_{n+1}\right|_{m}^{2} .
$$

Using Lemma 8.1 and the inequalities $\left|\nabla y_{n+1}\right|^{2} \leq 1, R<H^{2}$, we can estimate:

$$
\begin{aligned}
\left(\frac{\partial}{\partial t}-R^{p-1} \Delta_{m}\right) h & <2 k_{0}-\frac{4(n-1)}{n}\left[n-(n-\beta)\left|\nabla y_{n+1}\right|^{2}\right] H R^{p-1} \\
& \leq 2 k_{0}-\frac{4(n-1)}{n} \beta H R^{p-1}<2 k_{0}-\frac{4(n-1)}{n} \beta R^{p-\frac{1}{2}} .
\end{aligned}
$$

If $M_{t}$ does not remain enclosed by $B_{t}$ for all times under consideration, there exists a first time $\bar{t}$ at which $M_{\bar{t}}$ touches $B_{\bar{t}}$ from inside. If $\overline{\mathbf{p}}$ is a contact point, we have $h(\overline{\mathbf{p}}, \bar{t})=0$, while $h(\mathbf{p}, \mathbf{t}) \leq \mathbf{0}$ for $\mathbf{p} \in M$ and $t \leq \bar{t}$. Therefore we have

$$
\left(\frac{\partial}{\partial t}-R^{p-1} \Delta_{m}\right) h(\overline{\mathbf{p}}, \bar{t}) \geq 0 .
$$

On the other hand, the curvatures of $M_{\bar{t}}$ at a contact point are larger than the ones of $B_{\bar{t}}$. Since $R$ is increasing with respect to any $\lambda_{i}$ on the convex cone

$$
\left\{\left(\lambda_{1}, \ldots, \lambda_{n}\right) \in \mathbb{R}^{n}: H>0, R>0\right\}
$$


(see e.g. [17, Lemma 2.4]), we deduce that at a contact point also $R$ is larger on $M_{\bar{t}}$ than on $B_{\bar{t}}$. In particular, by (8.2), we deduce that $R(\overline{\mathbf{p}}, \bar{t}) \geq c_{0}$. Then (8.3) implies that

$$
\left(\frac{\partial}{\partial t}-R^{p-1} \Delta_{m}\right) h(\overline{\mathbf{p}}, \bar{t})<2 k_{0}-\frac{4(n-1)}{n} \beta c_{0}^{p-\frac{1}{2}} .
$$

If we choose

$$
k_{0}=\frac{2(n-1)}{n} \beta c_{0}^{p-\frac{1}{2}}
$$

we find a contradiction; therefore, for such a choice of $k_{0}$, the surfaces $M_{t}$ remain enclosed by the hyperboloids.

Since the hyperboloids $B_{t}$ converge to a cone as $t \rightarrow \eta^{2} / k_{0}$, the singular time of $M_{t}$ can be larger than this value only if at $t=\eta^{2} / k_{0}$ the surface $M_{t}$ is entirely contained in the half space $y_{n+1}>0$ or $y_{n+1}<0$. If we assume symmetry with respect to the hyperplane $y_{n+1}=0$ this is impossible; this proves the last part of the assertion.

Corollary 8.3. For any $n \geq 3$ and $p \geq 1 / 2$ there exists a closed nonconvex surface $M_{0} \subset \mathbb{R}^{n+1}$ with positive scalar curvature such that the corresponding solution of (1.1) does not shrink to a point as the singular time is approached.

Proof. A standard comparison argument with a shrinking sphere shows that, if $M_{0}$ encloses a sphere of radius $r_{0}$, then $M_{t}$ can only shrink to a point if its singular time is larger than the one of the sphere, which is equal to

$$
T^{*}\left(r_{0}\right)=\frac{r_{0}^{2 p+1}}{(2 p+1) n^{p}(n-1)^{p}} .
$$

Combining this property with Proposition 8.2, we can find examples of closed hypersurfaces which develop a neckpinch singularity for any dimension $n \geq 3$. We can take any $M_{0}$ which satisfies the properties of the proposition, including the symmetry with respect to the hyperplane $y_{n+1}=0$, and which encloses two spheres with a certain radius $r_{0}$ lying in the half space $y_{n+1}>0$ and $y_{n+1}<0$ respectively. Then, if the parameters are chosen in such a way that $\eta^{2} / k_{0}<T^{*}\left(r_{0}\right)$, we see that the evolving surface $M_{t}$ becomes singular at a time $T \leq \eta^{2} / k_{0}$ while still enclosing two spheres with positive radius.

It is easy to see that hypersurfaces $M_{0}$ satisfying the above requirements exist. One can fix the parameter $\beta \in(0, n-2)$ arbitrarily and pick any two spheres $B_{ \pm}$of radius $r_{0}$ centered at $\left(0, \pm L_{0}\right)$ for suitable $L_{0}$ which lie inside the cone $2|\mathbf{y}|^{2}-(n-$ $\beta) y_{n+1}^{2}=0$. In this way, the hyperboloid $2|\mathbf{y}|^{2}-(n-\beta) y_{n+1}^{2}=2 \eta^{2}$ also contains the two spheres for any $\eta>0$. We then set $N=L_{0}+r_{0}+1$, so that the two spheres are contained in the strip $-N+1 \leq y_{n+1} \leq N-1$. We consider the constant $k_{0}$ associated with $\beta, N$ by Proposition 8.2 and fix $\eta>0$ so that $\eta^{2} / k_{0}<T^{*}\left(r_{0}\right)$. It is easily checked that we can find a rotationally symmetric closed surface $M_{0}$ enclosing the spheres and contained both in the hyperboloid and in the strip. Since the hyperboloid has positive scalar curvature, it is also clear that such a surface can 
be chosen in such a way that it also has positive scalar curvature everywhere. An explicit construction can be found in [1].

\section{References}

[1] R. Alessandroni, "Evolution of Hypersurfaces by Curvature Functions", PhD Thesis, Università di Roma "Tor Vergata", 2008.

[2] B. ANDrEws, Contraction of convex hypersurfaces in Euclidean space, Calc. Var. Partial Differential Equations 2 (1994), 151-171.

[3] B. ANDREWS, Gauss curvature flow: the fate of rolling stones, Invent. Math. 138 (1999), $151-161$

[4] B. ANDREws, Motion of hypersurfaces by Gauss curvature, Pacific J. Math. 95 (2000), $1-36$.

[5] B. ANDREws, Fully nonlinear parabolic equations in two space variables, arXiv:math.AP/0402235 vl (2004).

[6] B. ANDREWs, Moving surfaces by non-concave curvature functions, Calc. Var. Partial Differential Equations, to appear.

[7] B. ANDREWS, Pinching estimates and motion of hypersurfaces by curvature functions, J. Reine Angew. Math. 608 (2007), 17-33.

[8] P. BAYARD, Entire scalar curvature flow and hypersurfaces of constant scalar curvature in Minkowski space, Methods Appl. Anal. 16 (2009), 87-118.

[9] B. CHOw, Deforming convex hypersurfaces by the nth root of the Gaussian curvature, J. Differential Geom. 22 (1985), 117-138.

[10] B. CHOw, Deforming convex hypersurfaces by the square root of the scalar curvature, Invent. Math. 87 (1987), 63-82.

[11] E. Di Benedetto and A. Friedman, Hölder estimates for nonlinear degenerate parabolic systems, J. Reine Angew. Math. 357 (1985), 1-22.

[12] K. ECKER, "Regularity Theory for Mean Curvature Flow", Birkhäuser, Boston, 2004.

[13] C. EnZ, The scalar curvature flow in lorentzian manifolds, Adv. Calc. Var. 1 (2008), 323343.

[14] G. Huisken, Flow by mean curvature of convex surfaces into spheres, J. Differential Geom. 20 (1984), 237-266.

[15] G. HUISKEN and A. POLDEN, Geometric evolution equation for hypersurfaces. In: "Calculus of Variations and Geometric Evolution Problems (CIME, Cetraro, 1996)", S. Hildebrandt et al. (eds.), Lect. Notes Math., Vol. 1713, Springer, Berlin, 1996, 45-84.

[16] G. HUISKEN and C. SinestraRI, Mean curvature flow singularities for mean convex surfaces, Calc. Var. Partial Differential Equations 8 (1999), 1-14.

[17] G. Huisken and C. Sinestrari, Convexity estimates for mean curvature flow and singularities of mean convex surfaces, Acta Math. 183 (1999), 45-70.

[18] N. V. KRYLOV, "Nonlinear Elliptic and Parabolic Equations of Second Order", D. Reidel, Dordrecht, 1987.

[19] G. M. Lieberman, "Second Order Parabolic Differential Equations", World Scientific, River Edge, NJ, 1996.

[20] O. C. SCHNÜRER, Surfaces contracting with speed $|A|^{2}$, J. Differential Geom. 71 (2005), 347-363.

[21] F. SCHULZE, Evolution of convex hypersurfaces by powers of the mean curvature, Math. Z. 251 (2005), 721-733.

[22] F. SCHUlzE, Convexity estimates for flows by powers of the mean curvature, with an appendix by O. C. Schnürer and F. Schulze, Ann. Scuola Norm. Sup. Pisa Cl. Sci. (5) 5 (2006), 261-277. 
[23] K. Tso, Deforming a hypersurface by its Gauss-Kronecker curvature, Comm. Pure Appl. Math. 38 (1985), 867-882.

Dipartimento di Matematica Università di Roma "Tor Vergata" Via della Ricerca Scientifica 00133 Roma, Italia alessand@mat.uniroma2.it. sinestra@mat.uniroma2.it. 